\title{
Birational Calabi-Yau threefolds and BPS state counting
}

\author{
YUKINOBU TODA
}

\begin{abstract}
This paper contains some applications of Bridgeland-Douglas stability conditions on triangulated categories, and Joyce's work on counting invariants of semistable objects, to the study of birational geometry. We introduce the notion of motivic Gopakumar-Vafa invariants as counting invariants of D2-branes, and show that they are invariant under birational transformations between Calabi-Yau threefolds. The result is similar to the fact that birational CalabiYau threefolds have the same betti numbers or Hodge numbers.
\end{abstract}

\section{Introduction}

First of all, let us recall the following well-known fact.

Theorem 1.1 [28]. Let $\phi: W \rightarrow X$ be a birational map between smooth projective Calabi-Yau threefolds. Then we have

$$
b_{i}(W)=b_{i}(X) \quad \text { for all } i \in \mathbb{Z} .
$$

Here $b_{i}(*)$ is the $i$-th betti number of $*$.

This result has been generalized for all dimensions by $[1,47]$ using the method of $p$-adic integration. Later on the equality of Hodge numbers $h^{p, q}(X)$ (more generally stringy Hodge numbers $h_{s t}^{p, q}(X)$ for varieties with log terminal singularities) under birational maps has been proved in $[2,10,32]$ using the method of motivic integration. (Also see $[19,48]$ for related works.)

In terms of string theory, the numbers $b_{i}(X), h^{p, q}(X)$ are interpreted as "counting invariants" of BPS D0-branes, which are mathematically stable zero-dimensional sheaves $\left\{\mathcal{O}_{x}\right\}_{x \in X}$. In this paper, we shall address the following question.

Question 1.2. How do counting invariants of BPS D2-branes (i.e., stable one-dimensional sheaves) transform under birational transformations? 
A similar problem has been studied in $[31,33,35,36]$ for Gromov-Witten invariants and in $[17,40]$ for Donaldson-Thomas invariants. In this paper, we are interested in Question 1.2 for Gopakumar-Vafa invariants, which were introduced by physicists Gopakumar and Vafa [13]. In [16], Hosono et al. proposed a first mathematical formulation of them. The purpose of this paper is to introduce another mathematical formulation which we call motivic Gopakumar-Vafa invariants and study their behavior under birational transformations. Our method is quite different from the works cited before, and uses Bridgeland-Douglas stability conditions on triangulated categories $[7,11,12]$, and Joyce's counting invariants of semistable objects [20-23].

\subsection{Gopakumar-Vafa invariants}

Let $X$ be a Calabi-Yau threefolds over $\mathbb{C}, \beta \in N_{1}(X)$ and $g \in \mathbb{Z}_{\geq 0}$, where $N_{1}(X)$ is the $\mathbb{R}$-vector space of numerical classes of one cycles. The 0 -point genus $g$ Gromov-Witten invariant of $X$ in numerical class $\beta$ is defined as the integration over the virtual fundamental class of the moduli space of stable $\operatorname{maps} \overline{\mathcal{M}}_{g, 0}(X, \beta)$,

$$
N_{g}^{\beta}:=\int_{\overline{\mathcal{M}}_{g, 0}^{\text {virt }}(X, \beta)} 1 \overline{\mathcal{M}}_{g, 0}(X, \beta) \in \mathbb{Q} .
$$

Although the invariants $N_{g}^{\beta}$ are not integers in general, Gopakumar and Vafa [13] claimed the following integrality of the generating function involv$\operatorname{ing} N_{g}^{\beta}$, based on the string duality between Type IIA and M-theory.

Conjecture 1.3. There are integers $n_{g}^{\beta, c o n j}$ and the following equality in $\mathbb{Q}\left[\left[N_{1}(X), \lambda^{ \pm 1}\right]\right]$

$$
\sum_{\beta, g} N_{g}^{\beta} q^{\beta} \lambda^{2 g-2}=\sum_{\beta, g, k} \frac{n_{g}^{\beta, c o n j}}{k}\left(2 \sin \left(\frac{k \lambda}{2}\right)\right)^{2 g-2} q^{k \beta} .
$$

They also claimed that the integer $n_{g}^{\beta, c o n j}$ should be defined by the "virtual counting of genus $g$ Jacobians" in the moduli space of the BPSbranes wrapping around holomorphic curves in $X$, and some computations are done in [26]. Its mathematical proposal by Hosono et al. [16] uses the relative Lefschetz action on the intersection cohomology of the moduli space 
of one-dimensional semistable sheaves $E$ with

$$
\left(\operatorname{ch}_{2}(E), \operatorname{ch}_{3}(E)\right)=(\beta, 1)
$$

which we denote by $M^{\beta}$. Using an $\mathfrak{s l}_{2} \times \mathfrak{s l}_{2}$-action on $I H^{*}\left(\widetilde{M}^{\beta}\right)$ where $\widetilde{M}^{\beta} \rightarrow M^{\beta}$ is the normalization, they defined an invariant $\tilde{n}_{g}^{\beta} \in \mathbb{Z}$ and conjectured that the invariants $\tilde{n}_{g}^{\beta}$ satisfy Conjecture 1.3.

However it seems that the invariants $\tilde{n}_{g}^{\beta}$ are unlikely to be deformation invariant, hence not equal to $n_{g}^{\beta \text {,cong }}$ exactly, since the definition of $\tilde{n}_{g}^{\beta}$ does not involve virtual classes. Now there is another approach of GopakumarVafa invariants using the notion of stable pairs, proposed by Pandharipande and Thomas [38].

\subsection{Main result}

Based on the work [16], we will construct invariants (cf. Definition 4.25)

$$
n_{g}^{\beta}(X) \in \mathbb{Z}, \quad \text { for } g \geq 0, \beta \in N_{1}(X),
$$

as a refinement of $\tilde{n}_{g}^{\beta}$. Roughly speaking $n_{g}^{\beta}(X)$ is defined using a certain motivic invariant, similar to the virtual Poincaré polynomial of $M^{\beta}$. Furthermore $n_{g}^{\beta}(X)$ is also defined for a non-effective one cycle class $\beta$. At least $n_{g}^{\beta}(X)$ coincides with $\tilde{n}_{g}^{\beta}$ if the moduli space $M^{\beta}$ is smooth and $\beta$ is represented by an effective one cycle. The following is our main theorem.

Theorem 1.4. Let $\phi: W \rightarrow X$ be a birational map between smooth projective Calabi-Yau threefolds. Then we have

$$
n_{g}^{\beta}(W)=n_{g}^{\phi_{*} \beta}(X),
$$

for all $g \geq 0$ and $\beta \in N_{1}(W)$.

It is worth mentioning that in the proof of Theorem 1.4, putting $\beta=0$ would result Theorem 1.1 (cf. Remark 5.7). The definition of $n_{g}^{\beta}$ also does not involve virtual classes, so we do not claim that $n_{g}^{\beta}$ satisfy Conjecture 1.3. However we have obtained a certain mathematical approximation of Gopakumar-Vafa invariants, which have birational invariance property.

\subsection{Strategy of the proof of Theorem 1.4}

We use the notion of stability conditions on triangulated categories introduced by T. Bridgeland [7], based on M. Douglas's work on M-stability 
$[11,12]$. Roughly speaking a stability condition on a triangulated category $\mathcal{D}$ consists of data

$$
Z: K(\mathcal{D}) \longrightarrow \mathbb{C}, \quad \mathcal{P}(\phi) \subset \mathcal{D},
$$

where $Z$ is a group homomorphism and $\mathcal{P}(\phi)$ is a subcategory for each $\phi \in \mathbb{R}$, which satisfy some axioms (Definition 3.1). We work over the triangulated category $\mathcal{D}=\mathcal{D}_{X}$ defined by

$$
\mathcal{D}_{X}:=\left\{E \in D^{b}(\operatorname{Coh}(X)) \mid \operatorname{dim} \operatorname{Supp}(E) \leq 1\right\}
$$

In terms of string theory, the set of objects $\{\mathcal{P}(\phi) \mid \phi \in \mathbb{R}\}$ is supposed to represent the set of BPS-branes at some point of the so-called stringy Kähler moduli space, the subspace of the moduli space of $\mathcal{N}=2$ super conformal field theories. Indeed Bridgeland [7] showed that the set of good stability conditions $\operatorname{Stab}(X)$ is a complex manifold, and expected that it describes the stringy Kähler moduli space mathematically. In this paper, we will construct a connected open subset (cf. Lemma 3.6),

$$
U_{X} \subset \operatorname{Stab}(X)
$$

which corresponds to the neighborhood of the large volume limit in string theory. Then our invariant $n_{g}^{\beta}(X)$ is defined as a certain counting invariant of objects $E \in \mathcal{P}(\phi)$ which satisfy (1.1) with respect to some point $(Z, \mathcal{P}) \in U_{X}$.

Next let us consider a birational map $\phi: W \rightarrow X$ from another CalabiYau threefolds $W$. Then due to Bridgeland [8], we have the equivalence of triangulated categories,

$$
\Phi: \mathcal{D}_{W} \longrightarrow \mathcal{D}_{X}
$$

which gives an isomorphism $\Phi_{*}: \operatorname{Stab}(W) \rightarrow \operatorname{Stab}(X)$. We claim that the closures of $\Phi_{*} U_{W}$ and $U_{X}$ intersect, in particular they are contained in the same connected component of $\operatorname{Stab}(X)$ (cf. Lemma 5.2). Then Question 1.2 is rephrased as follows.

Question 1.5. How do counting invariants vary by changing stability conditions, from $\sigma \in U_{X}$ to $\tau \in \Phi_{*} U_{W}$ ?

Now we use D. Joyce's theory on configurations on abelian categories and counting invariants of semistable objects [20-23]. Especially in [23], he studies how counting invariants of semistable objects vary under change of stability conditions. Although his works focus on stability conditions on abelian categories, his arguments also apply in our case. The reason 
is as follows: roughly speaking, a theory of stability conditions on abelian categories corresponds to a local theory on $\operatorname{Stab}(X)$. Thus Joyce's works enable us to study how counting invariants vary locally, and actually we will see they do not vary at all. Obviously we can answer Question 1.5, and conclude Theorem 1.4 by this argument.

The content of this paper is as follows. In Section 2 we review the mathematical proposal of Gopakumar-Vafa invariants in [16]. In Section 3 we review Bridgeland's work on stability conditions on triangulated categories [7], and construct some stability conditions we need. In Section 4 we introduce our invariant $n_{g}^{\beta}(X)$, and prove Theorem 1.4 in Section 5. In Section 6 we prove some technical lemmas.

\subsection{Terminology used in this paper}

In this paper, all the varieties are defined over $\mathbb{C}$. We say $X$ is Calabi-Yau if $X$ is smooth projective with trivial canonical bundle. For a variety $X$, we denote by $D(X)$ the derived category of coherent sheaves on $X$. For a triangulated category $\mathcal{D}$, its $K$-group is denoted by $K(\mathcal{D})$. We use the following standard terminology used in birational geometry [30],

$$
N^{1}(X):=\bigoplus_{D \subset X} \mathbb{R} D / \equiv, \quad N_{1}(X):=\bigoplus_{C \subset X} \mathbb{R} C / \equiv .
$$

In the definition of $N^{1}(X), D \subset X$ is a divisor and $D_{1} \equiv D_{2}$ if and only if $D_{1} \cdot C=D_{2} \cdot C$ for any curve $C$ on $X$. Similarly in the definition of $N_{1}(X)$, $C$ is a curve on $X$ and $C_{1} \equiv C_{2}$ if and only if $D \cdot C_{1}=D \cdot C_{2}$ for any divisor $D$. Cleary we have the perfect pairing,

$$
N^{1}(X) \times N_{1}(X) \ni(D, C) \longmapsto D \cdot C \in \mathbb{R}
$$

which identifies $N_{1}(X)$ with the dual of $N^{1}(X)$. We set $N^{1}(X)_{\mathbb{C}}=N^{1}(X)$ $\otimes_{\mathbb{R}} \mathbb{C}$ and

$$
\begin{aligned}
\overline{N E}(X) & :=\overline{\{\text { Cone of effective curves }\}} \subset N_{1}(X) \\
A(X)_{\mathbb{C}} & :=\left\{B+i \omega \in N^{1}(X)_{\mathbb{C}} \mid \omega \text { is ample }\right\} \subset N^{1}(X)_{\mathbb{C}} .
\end{aligned}
$$

Suppose that a birational map $\phi: W \rightarrow X$ is an isomorphism in codimension one. We use the following isomorphisms,

$$
\phi_{*}: N^{1}(W) \longrightarrow N^{1}(X), \quad \phi_{*}: N_{1}(W) \longrightarrow N_{1}(X),
$$


where the left-hand side is the strict transform and the right-hand side is the inverse of the dual of the LHS. For a non-zero $\beta \in N_{1}(X), \operatorname{Chow}_{\beta}(X)$ is the subvariety of the Chow variety Chow $(X)$, representing effective one cycles on $X$ with numerical class $\beta$. One can refer $[29$, chapter 1 , section $3]$ for the existence of the variety $\operatorname{Chow}_{\beta}(X)$. We set $\operatorname{Chow}_{\beta}(X)=\operatorname{Spec} \mathbb{C}$ when $\beta=0$. For a coherent sheaf $E$ on $X$ with $\operatorname{dim} \operatorname{Supp}(E) \leq 1$, we set

$$
s(E):=\sum_{p \in X} \operatorname{length}_{\mathcal{O}_{X, p}}\left(E_{p}\right) \overline{\{p\}} \in \operatorname{Chow}_{\beta}(X),
$$

where $\beta=\operatorname{ch}_{2}(E)$ and $p$ runs through all the codimension two points.

Let $X$ be a Calabi-Yau threefold. For an object $E \in \mathcal{D}_{X}$ and $v=$ $(\beta, k) \in N_{1}(X) \oplus \mathbb{Z}$, we say $E$ is of numerical type $v$ if

$$
\left(\operatorname{ch}_{2}(E), \operatorname{ch}_{3}(E)\right)=(\beta, k)
$$

\section{Review of work of Hosono et al.}

In this section, we briefly review the work of Hosono et al. [16].

\subsection{Representations of $\mathfrak{s l}_{2}$}

First let us recall that the Lie algebra $\mathfrak{s l}_{2}$ is generated by three elements,

$$
e=\left(\begin{array}{ll}
0 & 1 \\
0 & 0
\end{array}\right), \quad f=\left(\begin{array}{ll}
0 & 0 \\
1 & 0
\end{array}\right), \quad h=\left(\begin{array}{rr}
1 & 0 \\
0 & -1
\end{array}\right)
$$

which satisfy the relation,

$$
[e, f]=h, \quad[h, e]=2 e, \quad[h, f]=-2 f .
$$

For each $j \in \frac{1}{2} \mathbb{Z}$, there is a unique irreducible representation of $\mathfrak{s l}_{2}$ (up to isomorphism) of dimension $2 j+1$, called the spin $j$ representation, and denoted by $(j)$. For $V=(j)$, there is an eigenvector $v \in V$ of $h$ such that $f v=0$ and

$$
(j)=<v, e v, \ldots, e^{2 j} v>, \quad e^{2 j+1} v=0,
$$

with $h e^{k} v=(-2 j+2 k) v, 0 \leq k \leq 2 j$.

Let $X$ be a normal projective variety and $I H^{*}(X)$ is the intersection cohomology of $X$ introduced in [4]. Note that if $X$ is connected and smooth, 
we have

$$
I H^{i}(X)=H^{i+\operatorname{dim} X}(X, \mathbb{C}),
$$

for any $i \in \mathbb{Z}$. Let $H$ be an ample divisor on $X$, and $\eta$ be the Lefschetz operator,

$$
\eta=H \wedge: I H^{*}(X) \longrightarrow I H^{*+2}(X) .
$$

It is well known that the operator $\eta^{i}: I H^{-i}(X) \rightarrow I H^{i}(X)$ is an isomorphism. (cf. [4, theorems 5.4.10 and 6.2.10].) Using this, one can construct an $\mathfrak{s l}_{2}$-action on $I H^{*}(X)$ as follows (see [39, section 2] for details). First we find a homogeneous basis of $I H^{*}(X)$ which consist of primitive elements. Here $v \in I H^{-i}(X)$ for $i \geq 0$ is primitive if $\eta^{i+1} v=0$. For such a basis $\left\{v_{\alpha}\right\}$, $I H^{*}(X)$ is written as a direct sum of the subspaces generated by

$$
\left\{v_{\alpha}, \eta v_{\alpha}, \ldots, \eta^{i_{\alpha}} v_{\alpha}\right\}
$$

with $\operatorname{deg} v_{\alpha}=-i_{\alpha}$. Then define the representation of $\mathfrak{s l}_{2}$ on $I H^{*}(X)$ by letting $e \in \mathfrak{s l}_{2}$ act as $\eta, h \in \mathfrak{s l}_{2}$ act as multiplying by the degree and the action of $f \in \mathfrak{s l}_{2}$ is defined inductively from the requirement of $f v_{\alpha}=0$. Hence the subspace (2.1) gives spin $\left(\frac{i_{\alpha}}{2}\right)$-representation. For a complex torus $T$ of dimension $g$, we have the following formula (see [16, section 2]).

$$
I H^{*}(T)=I_{g}:=\left[\left(\frac{1}{2}\right) \oplus 2(0)\right]^{\otimes g} .
$$

Lemma 2.1. The $\mathfrak{s l}_{2}$-representation type of $I H^{*}(X)$ does not depend on a choice of an ample divisor $H$.

Proof. Let $H^{\prime}$ be another ample divisor, and consider the operator $\eta^{\prime}(*)=$ $H^{\prime} \wedge *$. Let $T_{j}, T_{j}^{\prime} \subset I H^{*}(X)$ be the sub $\mathfrak{s l}_{2}$-representations with respect to the operators $\eta, \eta^{\prime}$ respectively, consisting of direct sums of spin $\left(j^{\prime}\right)$ representations for $j^{\prime} \geq j$. Suppose that $T_{j}, T_{j}^{\prime}$ have the same $\mathfrak{s l}_{2}$-represention types. Then the minimal degrees of the following graded vector spaces,

$$
I H^{*}(X) / T_{j}, \quad I H^{*}(X) / T_{j}^{\prime},
$$

are same, say $d \in(-2 j, 0]$. Also the subspaces of degree $d$ elements in $(2.2)$ have the same dimensions, say $l$. Then we see

$$
T_{-d / 2} \cong T_{j} \oplus\left(-\frac{d}{2}\right)^{\oplus l}, \quad T_{-d / 2}^{\prime} \cong T_{j}^{\prime} \oplus\left(-\frac{d}{2}\right)^{\oplus l}
$$


as $\mathfrak{s l}_{2}$-representations. Therefore $T_{-d / 2}$ and $T_{-d / 2^{\prime}}$ have the same $\mathfrak{s l}_{2}$-representation types. By the induction we obtain the lemma.

\subsection{Relative Lefschetz actions}

Let $f: X \rightarrow A$ be a projective morphism between normal projective varieties. The idea of [16] is to define the $\left(\mathfrak{s l}_{2}\right)_{L} \times\left(\mathfrak{s l}_{2}\right)_{R}$-action on $I H^{*}(X)$ using the Lefschetz operators in fiber directions and base directions. Let $H_{A}, H_{X / A}$ be an ample divisor on $A$, a relative ample divisor on $X$ over $A$ respectively. We denote by $D\left(\mathbb{C}_{X}\right)$, Perv $\left(\mathbb{C}_{X}\right)$ the derived category of constructible sheaves on $X$ (with its classical topology), the heart of the middle perverse $t$-structure on $D\left(\mathbb{C}_{X}\right)$ respectively. We have the perverse Leray spectral sequence,

$$
E_{2}^{r, s}=H^{r}\left(A,{ }^{p} R^{s} f_{*} \mathcal{I} C_{X}\right) \Longrightarrow I H^{r+s}(X),
$$

where $\mathcal{I} C_{X} \in \operatorname{Perv}\left(\mathbb{C}_{X}\right)$ is the intersection complex on $X$, and ${ }^{p} R^{s} f_{*} \mathcal{I} C_{X} \in$ $\operatorname{Perv}\left(\mathbb{C}_{A}\right)$ is the $s$-th cohomology of $\mathbf{R} f_{*} \mathcal{I} C_{X}$ with respect to the middle perverse $t$-structure on $D\left(\mathbb{C}_{A}\right)$. It is known that this spectral sequence degenerates at $E_{2}$-terms (cf. [4, theorem 6.2 .5$]$ ), and we have two operators,

$$
\eta_{L}=H_{X / A} \wedge: E_{2}^{r, s} \longrightarrow E_{2}^{r, s+2}, \quad \eta_{R}=H_{A} \wedge: E_{2}^{r, s} \longrightarrow E_{2}^{r+2, s}
$$

such that $\eta_{L}^{s}: E_{2}^{r,-s} \cong E_{2}^{r, s}$ and $\eta_{R}^{r}: E_{2}^{-r, s} \cong E_{2}^{r, s}$. As in Paragraph 2.1, these two actions define an $\left(\mathfrak{s l}_{2}\right)_{L} \times\left(\mathfrak{s l}_{2}\right)_{R}$-action on $I H^{*}(X)$ (cf. [16, corollary 2.1]). Also by the same argument of Lemma 2.1 , the $\left(\mathfrak{s l}_{2}\right)_{L} \times\left(\mathfrak{s l}_{2}\right)_{R^{-}}$ representation type of $I H^{*}(X)$ does not depend on $H_{X / A}, H_{A}$.

\subsection{Definition of HST (Hosono, Saito, Takahashi) invariants}

Let $X$ be a projective variety. For $\beta \in N_{1}(X)$ and an ample divisor $H$ on $X$, let $M^{\beta}$ be the moduli space of $H$-Gieseker semistable sheaves $E$ on $X$ (cf. [18]), pure of dimension one, with numerical type $(\beta, 1)$. Let $\widetilde{M}^{\beta} \rightarrow M^{\beta}$ be the normalization. By the same argument as in $[37$, chapter 5 , section 4], there is a natural map,

$$
\pi_{\beta}: M^{\beta} \ni E \longmapsto s(E) \in \operatorname{Chow}_{\beta}(X)
$$

and let $S^{\beta}$ the normalization of the image of $\pi_{\beta}$. The induced morphism $\pi_{\beta}: \widetilde{M}^{\beta} \rightarrow S^{\beta}$ is projective, hence defines an $\left(\mathfrak{s l}_{2}\right)_{L} \times\left(\mathfrak{s l}_{2}\right)_{R}$-action on 
$I H^{*}\left(\widetilde{M}^{\beta}\right)$. One can rearrange its action in the following formula (cf. $[16$, theorem 2.4]),

$$
I H^{*}\left(\widetilde{M}^{\beta}\right)=\bigoplus_{g \geq 0} I_{g} \otimes R_{g}(\beta),
$$

where $R_{g}(\beta)$ is a virtual $\left(\mathfrak{s l}_{2}\right)_{R}$-representation.

Definition 2.2 [16, definition 3.6]. We define $\tilde{n}_{g}^{\beta}$ to be

$$
\tilde{n}_{g}^{\beta}=\sum_{j}(-1)^{2 j}(2 j+1) \cdot N_{j} \in \mathbb{Z}
$$

after writing $R_{g}(\beta)=\sum_{j} N_{j} \cdot(j)_{R}$ as a virtual representation.

Remark 2.3. In [16, definition 3.6], the invariant $\tilde{n}_{g}^{\beta}$ is defined as $\operatorname{Tr}_{R_{g}(\beta)}$ $(-1)^{h_{R}}$, which coincides with formula (2.3).

Remark 2.4. As pointed out in [38], the invariants $\tilde{n}_{g}^{\beta}$ are unlikely to be BPS-invariants discussed in [13]. We need to involve virtual classes to define appropriate BPS-counting which are deformation invariant.

There is an alternative way of defining $\tilde{n}_{g}^{\beta}$ pointed out by [39], and it is much more useful for our purposes. Let $V$ be the space of an $\left(\mathfrak{s l}_{2}\right)_{L} \times\left(\mathfrak{s l}_{2}\right)_{R^{-}}$ representation. Then the operator $h_{L}+h_{R}$ defines the grading $V=\oplus V_{n}$ with $e_{R} V_{n} \subset V_{n+2}$. We can decompose $V$ into the direct sum of the vector subspaces spanned by

$$
v, e_{R} v, \ldots, e_{R}^{l-1} v,
$$

where $v \in V_{\alpha}$ for some $\alpha, e_{R}^{l} v=0$, and there is no $v^{\prime} \in V$ with $e_{R} v^{\prime}=v$. Such a subspace is called a Jordan cell of size $l$ and minimal degree $\alpha$. Let $\nu_{l}^{\alpha} \in \mathbb{Z}_{\geq 0}$ be

$$
\nu_{l}^{\alpha}=\sharp\{\text { Jordan cells of size } l \text { and minimal degree } \alpha \text { in } V\} .
$$

Note that $\nu_{l}^{\alpha}$ depends only on the $e_{R}$-action and the grading induced by $h_{L}+h_{R}$. We have the following.

Proposition 2.5 [39]. For $V=I H^{*}\left(\widetilde{M}^{\beta}\right)$, we have

$$
\tilde{n}_{g}^{\beta}=\sum_{\alpha+l \geq 1}(-1)^{\alpha+g} l \nu_{l}^{\alpha}\left\{\left(\begin{array}{c}
\alpha+l+g \\
2 g+1
\end{array}\right)-\left(\begin{array}{c}
\alpha+l+g-2 \\
2 g+1
\end{array}\right)\right\} .
$$




\section{Stability conditions on triangulated categories}

In this section we briefly recall the notion of stability conditions on triangulated categories [7], fix some notation and prove some fundamental properties.

\subsection{Generalities}

The notion of stability conditions on a triangulated category $\mathcal{D}$ was introduced by T. Bridgeland [7] motivated by M. Douglas's work on $\Pi$-stability $[11,12]$. Here we only introduce its definition and some terminology used in this paper, and do not explain its review too much. For the readers who are not familiar with [7], we recommend consulting the original paper [7].

Definition 3.1. A stability condition on $\mathcal{D}$ consists of data $\sigma=(Z, \mathcal{P})$,

$$
Z: K(\mathcal{D}) \longrightarrow \mathbb{C}, \quad \mathcal{P}(\phi) \subset \mathcal{D}
$$

where $Z$ is a group homomorphism, $\mathcal{P}(\phi)$ is a subcategory for each $\phi \in \mathbb{R}$ which satisfies,

- $\mathcal{P}(\phi+1)=\mathcal{P}(\phi)[1]$.

- If $\phi_{1}>\phi_{2}$ and $A_{i} \in \mathcal{P}\left(\phi_{i}\right)$, then $\operatorname{Hom}\left(A_{1}, A_{2}\right)=0$.

- If $E \in \mathcal{P}(\phi)$ is non-zero, then $Z(E)=m(E) \exp (i \pi \phi)$ for some $m(E) \in$ $\mathbb{R}_{>0}$.

- For a non-zero object $E \in \mathcal{T}$, we have the following collection of triangles:

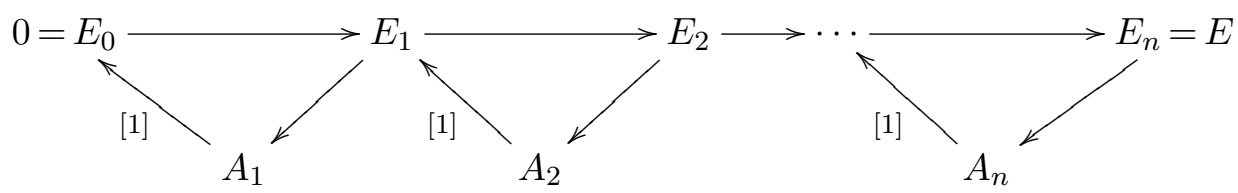

such that $A_{j} \in \mathcal{P}\left(\phi_{j}\right)$ with $\phi_{1}>\phi_{2}>\cdots>\phi_{n}$.

A non-zero object in $\mathcal{P}(\phi)$ is called semistable of phase $\phi$. The mass of $E$ is defined to be

$$
m_{\sigma}(E)=\sum_{j}\left|Z\left(A_{j}\right)\right| .
$$


For an interval $I \subset \mathbb{R}$, we denote by $\mathcal{P}(I)$ the smallest extension closed subcategory of $\mathcal{D}$ which contains $\mathcal{P}(\phi)$ with $\phi \in I$. It is easy to see that $\mathcal{P}((0,1])$ is the heart of a bounded $t$-structure on $\mathcal{D}$. This gives an alternative way of constructing a stability condition.

Proposition 3.2 [7, proposition 4.2]. Giving a stability condition is equivalent to giving the heart of a bounded t-structure $\mathcal{A} \subset \mathcal{D}$ together with a group homomorphism $Z: K(\mathcal{A}) \rightarrow \mathbb{C}$ such that for a non-zero object $E \in \mathcal{A}$ one has

$$
Z(E) \in \mathbb{H}:=\{r \exp (i \pi \phi) \mid r>0,0<\phi \leq 1\}
$$

and the pair $(Z, \mathcal{A})$ satisfies the Harder-Narasimhan property.

The set of stability conditions which satisfy the local finiteness (cf. [7, definition 5.7]) is denoted by $\operatorname{Stab}(\mathcal{D})$. It is shown in $[7$, section 6] that $\operatorname{Stab}(\mathcal{D})$ has a natural topology. Furthermore for each connected component $\Sigma \subset \operatorname{Stab}(\mathcal{D})$, there exists a linear subspace $V(\Sigma) \subset \operatorname{Hom}_{\mathbb{Z}}(K(\mathcal{D}), \mathbb{C})$ with a norm such that we have the local homeomorphism (cf. [7, theorem 1.2]),

$$
\mathcal{Z}: \Sigma \ni(Z, \mathcal{P}) \longmapsto Z \in V(\Sigma) \text {. }
$$

\subsection{Stability conditions on Calabi-Yau threefolds}

For a Calabi-Yau threefold $X$, we consider the following triangulated category as in the introduction,

$$
\mathcal{D}_{X}:=\{E \in D(X) \mid \operatorname{dim} \operatorname{Supp}(E) \leq 1\}
$$

Here we introduce the subspace of $\operatorname{Stab}\left(\mathcal{D}_{X}\right)$, coming from the points corresponding to the neighborhood of the large volume limit at $X$. For $B+i \omega \in$ $N^{1}(X)_{\mathbb{C}}$, we set $Z_{(B, \omega)}: K\left(\mathcal{D}_{X}\right) \rightarrow \mathbb{C}$ as

$$
Z_{(B, \omega)}(E)=-\operatorname{ch}_{3}(E)+(B+i \omega) \cdot \operatorname{ch}_{2}(E)
$$

Remark 3.3. Note that $Z_{(B, \omega)}$ factors as follows,

$$
Z_{(B, \omega)}: K\left(\mathcal{D}_{X}\right) \stackrel{\left(\mathrm{ch}_{2}, \mathrm{ch}_{3}\right)}{\longrightarrow} N_{1}(X) \oplus \mathbb{Z} \longrightarrow \mathbb{C}
$$

Here the right arrow takes $v=(\beta, k)$ to $-k+(B+i \omega) \beta$, which we write as $Z_{(B, \omega)}(v)$ by abuse of notation. 
We also set $\operatorname{Coh}_{\leq 1}(X)$ as $\operatorname{Coh}_{\leq 1}(X):=\operatorname{Coh}(X) \cap \mathcal{D}_{X}$. Note that $\operatorname{Coh}_{\leq 1}$ $(X)$ is the heart of a bounded t-structure on $\mathcal{D}_{X}$. We have the following lemma.

Lemma 3.4. For $B+i \omega \in A(X)_{\mathbb{C}}$, the pair

$$
\sigma_{(B, \omega)}:=\left(Z_{(B, \omega)}, \operatorname{Coh}_{\leq 1}(X)\right)
$$

determines a point in $\operatorname{Stab}\left(\mathcal{D}_{X}\right)$ in the sense of Proposition 3.2.

Proof. The proof of this lemma is exactly same as in [42, lemma 4.1]. In fact for a non-zero $E \in \mathrm{Coh}_{\leq 1}(X)$, we have $\operatorname{Im} Z_{(B, \omega)}(E)>0$ when $\operatorname{dim} \operatorname{Supp}$ $(E)=1$ and $Z_{(B, \omega)}(E) \in \mathbb{R}_{<0}$ when $\operatorname{dim} \operatorname{Supp}(E)=0$. Thus (3.1) holds. One can also check the Harder-Narasimhan property as in [42, lemma 4.1].

Remark 3.5. For an object $E \in \mathrm{Coh}_{\leq 1}(X)$, it is $\sigma_{(0, \omega)}$-semistable if and only if for any non-trivial subobject $F \subset E$ in $\operatorname{Coh}_{\leq 1}(X)$, one has

$$
\frac{\operatorname{ch}_{3}(F)}{\omega \cdot \operatorname{ch}_{2}(F)} \leq \frac{\operatorname{ch}_{3}(E)}{\omega \cdot \operatorname{ch}_{2}(E)},
$$

i.e., $E$ is $\omega$-Gieseker semistable sheaf.

We define $\operatorname{Stab}(X)$ to be the following fiber product,

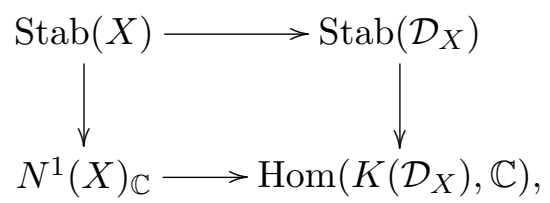

where the right arrow takes $(Z, \mathcal{P})$ to $Z$ and the bottom arrow takes $B+i \omega$ to $Z_{(B, \omega)}$. Note that the stability conditions constructed in Lemma 3.4 are contained in $\operatorname{Stab}(X)$. Let $U_{X} \subset \operatorname{Stab}(X)$ be

$$
U_{X}:=\left\{\sigma_{(B, \omega)} \in \operatorname{Stab}(X) \mid B+i \omega \in A(X)_{\mathbb{C}}\right\} .
$$

We have to check the following, whose proof will be postponed in Section 6 . 
Lemma 3.6. The subset $U_{X}$ is an open connected subset of $\operatorname{Stab}(X)$.

By [7, theorem 1.1] and Lemma 3.6, the map

$$
\mathcal{Z}_{X}: \operatorname{Stab}(X) \ni(Z, \mathcal{P}) \longmapsto Z \in N^{1}(X)_{\mathbb{C}}
$$

restricts to a homeomorphism between $U_{X}$ and $A(X)_{\mathbb{C}}$.

\subsection{Wall and chamber structures}

In this paragraph, we recall the notion of wall and chamber structures on the space of stability conditions. The wall and chamber structure is introduced in [6] on the space of stability conditions on K3 surfaces, and we show that our space $\operatorname{Stab}(X)$ also possesses such a structure. Let

$$
\operatorname{Stab}^{*}(X) \subset \operatorname{Stab}(X)
$$

be the connected component which contains $U_{X}$. Let $\mathcal{S} \subset \mathcal{D}_{X}$ be a set of objects. For $\sigma \in \operatorname{Stab}^{*}(X)$, we call $\mathcal{S}$ has bounded mass if there is $m>0$ such that

$$
m_{\sigma}(E)<m,
$$

for any $E \in \mathcal{S}$. It is easy to show that if this condition holds for $\sigma \in$ $\operatorname{Stab}^{*}(X)$, then it also holds for any $\sigma^{\prime} \in \operatorname{Stab}^{*}(X)$. We have the following proposition (cf. [6, proposition 9.3]).

Proposition 3.7. For a fixed compact subset $\overline{\mathcal{U}} \subset \operatorname{Stab}^{*}(X)$, there is a finite number of real codimension one submanifolds $\left\{\mathcal{W}_{\gamma} \mid \gamma \in \Gamma\right\}$ such that each connected component

$$
\mathcal{C} \subset \overline{\mathcal{U}} \backslash \bigcup_{\gamma \in \Gamma} \mathcal{W}_{\gamma}
$$

has the following property. If $E \in \mathcal{S}$ is $\sigma$-semistable for some $\sigma \in \mathcal{C}$, then $E$ is semistable in $\sigma^{\prime}$ for all $\sigma^{\prime} \in \mathcal{C}$.

Proof. The same proof of [6, proposition 9.3] applies once we show the analogue of [6, lemma 9.2] in our case, i.e., the set of numerical classes,

$$
\left\{\left(\operatorname{ch}_{2}(E), \operatorname{ch}_{3}(E)\right) \mid E \in \mathcal{S}\right\} \subset N_{1}(X) \oplus \mathbb{Z},
$$

is a finite set. For an ample divisor $\omega$, let us take $\sigma=\sigma_{(0, \omega)}$. Also for $E \in \mathcal{S}$, let $A_{1}, \ldots, A_{n}$ be the $\sigma$-semistable factors. Then the bounded mass 
condition for $\mathcal{S}$ implies that the values

$$
\left|\operatorname{ch}_{3}\left(A_{i}\right)\right|, \quad\left|\operatorname{ch}_{2}\left(A_{i}\right) \cdot \omega\right|
$$

are bounded. On the other hand, the following space,

$$
\{c \in \overline{N E}(X) \mid c \cdot \omega \leq 1\} \subset N_{1}(X)
$$

is compact. In fact openness of the ample cone immediately implies the compactness of the earlier space. Since $\operatorname{ch}_{2}\left(A_{i}\right)$ or $-\operatorname{ch}_{2}\left(A_{i}\right)$ is contained in $\overline{N E}(X)$, we see that the pair $\left(\operatorname{ch}_{2}\left(A_{i}\right), \operatorname{ch}_{3}\left(A_{i}\right)\right)$ has finite number of possibilities. Hence (3.2) is also finite.

We call a connected component $\mathcal{C} \subset \overline{\mathcal{U}} \backslash \bigcup_{\gamma \in \Gamma} \mathcal{W}_{\gamma}$ chamber

\subsection{Moduli theory of semistable objects}

In this paragraph, we consider the moduli problem of $\sigma$-semistable objects $E \in \mathcal{D}_{X}$ for $\sigma \in \operatorname{Stab}(X)$. Such a moduli theory is studied in [41] for stability conditions on K3 surfaces and abelian surfaces, and we use some basic arguments developed there. Let $\mathcal{M}$ be the moduli stack of objects $E \in \mathcal{D}_{X}$, satisfying the following condition,

$$
\operatorname{Ext}^{i}(E, E)=0, \quad \text { for } i<0 \text {. }
$$

Then by the result of Lieblich [34], the stack $\mathcal{M}$ is an Artin stack of locally finite type over $\mathbb{C}$. More precisely, Lieblich showed that the stack of $E \in$ $D(X)$ satisfying the previous condition is an Artin stack of locally finite type. However for a family of objects $\mathcal{E} \in D(X \times S)$, the condition $\mathcal{E}_{s} \in \mathcal{D}_{X}$ is obviously an open condition, thus $\mathcal{M}$ is also an Artin stack of locally finite type.

For $\sigma=(Z, \mathcal{P}) \in \bar{U}_{X}, v \in N_{1}(X) \oplus \mathbb{Z}$ and $\phi \in \mathbb{R}$, we can consider the substack,

$$
i: \mathcal{M}^{(v, \phi)}(\sigma) \hookrightarrow \mathcal{M}
$$

which is the moduli stack of $E \in \mathcal{P}(\phi)$ of numerical type $v$. The purpose here is to show that $\mathcal{M}^{(v, \phi)}(\sigma)$ is algebraic. Recall that a set of objects $\mathcal{S} \subset \mathcal{D}_{X}$ is called bounded if there is a finite type $\mathbb{C}$-scheme $S$ together with an object $\mathcal{E} \in D(X \times S)$ such that any object $E \in \mathcal{S}$ is isomorphic to $\mathcal{E}_{s}$ for some $s \in S$. We use the following lemma, whose proof will be postponed until Section 6. 
Lemma 3.8. Let us take $\sigma=(Z, \mathcal{P}) \in \operatorname{Stab}^{*}(X)$ such that $Z$ is defined over $\mathbb{Q}$. Then the set of objects,

$$
\mathcal{M}^{(v, \phi)}(\sigma)(\operatorname{Spec} \mathbb{C})=\{E \in \mathcal{P}(\phi) \mid E \text { is of numerical type } v\}
$$

is bounded.

Using this lemma and the argument in [41], we show the following proposition.

Proposition 3.9. The stack $\mathcal{M}^{(v, \phi)}(\sigma)$ is an Artin stack of finite type over $\mathbb{C}$, and $i: \mathcal{M}^{(v, \phi)}(\sigma) \hookrightarrow \mathcal{M}$ is an open immersion.

Proof. For $B$ and $\omega$ are rational, the set of $\mathbb{C}$-valued points of $\mathcal{M}^{(v, \phi)}\left(\sigma_{(B, \omega)}\right)$ is bounded by Lemma 3.8. Hence by [41, lemma 3.13 (ii), proposition 3.18] the result is true for such $\sigma_{(B, \omega)}$. Then one can apply [41, theorem 3.20, step 1], and conclude the result for any $\sigma \in \bar{U}_{X}$.

\section{Motivic Gopakumar-Vafa invariants}

The purpose of this section is to introduce the invariants $n_{g}^{\beta}(X) \in \mathbb{Z}$, from a certain motivic invariant of varieties over a Chow variety.

\subsection{Motivic invariants of varieties}

Let $A$ be a projective variety over $\mathbb{C}$. First let us recall the Grothendieck group of varieties over $A$.

Definition 4.1. We define the $\mathbb{Z}$-module $K_{0}(\operatorname{Var} / A)$ to be

$$
K_{0}(\operatorname{Var} / A)=\bigoplus \mathbb{Z}[(X, \pi)] / \sim
$$

where $[(X, \pi)]$ is an isomorphism class of a pair of a quasi-projective variety $X$ together with a morphism $\pi: X \rightarrow A$. The equivalence relation $\sim$ is generated by

$$
[(X, \pi)]=\left[\left(Z,\left.\pi\right|_{Z}\right)\right]+\left[\left(X \backslash Z,\left.\pi\right|_{X \backslash Z)}\right),\right.
$$

for closed subvarieties $Z \subset X$.

Let $\pi: X \rightarrow A$ be a projective morphism with $X$ smooth and connected. There is an induced morphism $X \rightarrow \widetilde{A}$, where $\widetilde{A}$ is the normalization of 
$\pi(A)$. Then as in Paragraph 2.2, IH $H^{*}(X)$ carries an $\left(\mathfrak{s l}_{2}\right)_{L} \times\left(\mathfrak{s l}_{2}\right)_{R^{-a c t i o n}}$ with respect to the morphism $X \rightarrow \widetilde{A}$. Let $\nu_{l}^{\alpha} \in \mathbb{Z}$ be the number of Jordan cells in $I H^{*}(X)$ for this action, defined in (2.4). We set $P(X, \pi) \in \mathbb{Z}[t, s]$ as follows:

$$
P(X, \pi)=t^{\operatorname{dim} X} \sum_{\alpha, l} \nu_{l}^{\alpha} t^{\alpha} s^{l-1} .
$$

We show the following proposition.

Proposition 4.2. There exists a map,

$$
\Upsilon_{A}: K_{0}(\operatorname{Var} / A) \longrightarrow \mathbb{Z}[t, s],
$$

such that for any projective morphism $\pi: X \rightarrow A$ with $X$ smooth and connected, we have

$$
\Upsilon_{A}([(X, \pi)])=P(X, \pi)
$$

Proof. Let $X$ be a connected smooth projective variety with a morphism $\pi: X \rightarrow A$. Let $Z \subset X$ be a smooth closed subvariety and take the blow-up $p: X^{\dagger} \rightarrow X$ along $Z$,

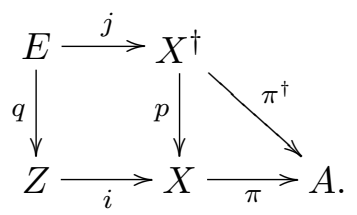

By the result of [5, theorem 5.1], $K_{0}(\operatorname{Var} / A)$ is generated by such $[(X, \pi)]$ with relation

$$
\left[\left(X^{\dagger}, \pi^{\dagger}\right)\right]-\left[\left(E, \pi^{\dagger} \circ j\right)\right]=[(X, \pi)]-[(Z, \pi \circ i)] .
$$

Hence it is enough to show that

$$
P\left(X^{\dagger}, \pi^{\dagger}\right)-P\left(E, \pi^{\dagger} \circ j\right)=P(X, \pi)-P(Z, \pi \circ i) .
$$

Let $d=\operatorname{dim} X$ and $r$ be the codimension of $Z$ in $X$. Note that $q: E \rightarrow Z$ is a $\mathbb{P}^{r-1}$-bundle. By the isomorphism (4.3) in Lemma 4.3 next, we have

$$
\frac{P\left(X^{\dagger}, \pi^{\dagger}\right)}{t^{d}}=\frac{P(X, \pi)}{t^{d}}+\frac{t^{-r+2} P(Z, \pi \circ i)}{t^{d-r}}+\cdots+\frac{t^{r-2} P(Z, \pi \circ i)}{t^{d-r}},
$$


since $\mu_{l}^{\alpha}$ is determined by the grading and $e_{R}$-actions. Thus we have

$$
P\left(X^{\dagger}, \pi^{\dagger}\right)=P(X, \pi)+t^{2} P(Z, \pi \circ i)+\cdots+t^{2(r-1)} P(Z, \pi \circ i) .
$$

Similarly the isomorphism (4.4) in Lemma 4.3 yields,

$$
P\left(E, \pi^{\dagger} \circ j\right)=P(Z, \pi \circ i)+t^{2} P(Z, \pi \circ i)+\cdots+t^{2(r-1)} P(Z, \pi \circ i) .
$$

Hence the Equation (4.2) holds.

We have used the following lemma.

Lemma 4.3. In the diagram (4.1), we have the following isomorphisms,

$$
\begin{aligned}
I H^{*}\left(X^{\dagger}\right) & \cong I H^{*}(X) \oplus \bigoplus_{i=0}^{r-2} I H^{*}(Z)[-r+2+2 i], \\
I H^{*}(E) \cong & \bigoplus_{i=0}^{r-1} I H^{*}(Z)[-r+1+2 i]
\end{aligned}
$$

which preserve $e_{R}$-actions.

Proof. Let $\mathcal{O}_{E}(1)$ be the tautological line bundle on $E$ and set $\xi=c_{1}\left(\mathcal{O}_{E}(1)\right)$ $\in H^{2}(E, \mathbb{C})$. We have the following isomorphism,

$$
H^{*}(Z, \mathbb{C}) \oplus H^{*}(Z, \mathbb{C})[-2] \oplus \cdots \oplus H^{*}(Z, \mathbb{C})[-2(r-1)] \stackrel{\cong}{\longrightarrow} H^{*}(E, \mathbb{C}),
$$

which sends $\left(v_{0}, v_{1}, \ldots, v_{r-1}\right)$ to $\sum \xi^{i} q^{*} v_{i}$. It is obvious that (4.5) preserves $e_{R}$-actions, hence (4.4) follows.

Next in $[14$, p. 605], it is shown that we have the isomorphism,

$$
H^{*}\left(X^{\dagger}, \mathbb{C}\right) \stackrel{\cong}{\longrightarrow} H^{*}(X, \mathbb{C}) \oplus \frac{H^{*}(E, \mathbb{C})}{q^{*} H^{*}(Z, \mathbb{C})},
$$

which sends $v \in H^{*}(X, \mathbb{C})$ to $\left(p_{*} v, j^{*} v\right)$. For an ample divisor $H_{A}$ on $A$, we have

$$
\pi^{*} H_{A} \wedge p_{*} v=p_{*}\left(p^{*} \pi^{*} H_{A} \wedge v\right), \quad j^{*} \pi^{\dagger *} H_{A} \wedge j^{*} v=j^{*}\left(\pi^{\dagger *} H_{A} \wedge v\right) .
$$

Hence the isomorphism (4.6) preserves $e_{R}$-actions. Using (4.4) and (4.6), we obtain the isomorphism (4.3).

In the following, we compute some examples of our nvariant $P(X, \pi)$. 
Example 4.4. (i) Let $A$ be a $d$-dimensional smooth projective variety and consider the element $[(A, \mathrm{id})] \in K_{0}(\operatorname{Var} / A)$. Let $b_{i}(A) \in \mathbb{Z}$ be the $i$-th betti number of $A$. Then one can easily see

$$
\nu_{l}^{\alpha}=\left\{\begin{array}{cl}
b_{d+\alpha}(A)-b_{d+\alpha-2}(A), & \text { if } l=-\alpha+1 \\
0, & \text { otherwise }
\end{array}\right.
$$

Hence we have

$$
P(A, \mathrm{id})=\sum_{\alpha=0}^{d}\left(b_{\alpha}(A)-b_{\alpha-2}(A)\right) t^{\alpha} s^{-\alpha+d} .
$$

(ii) Let $\pi: X \rightarrow A$ be a projective bundle with fiber $\mathbb{P}^{r-1}$. Then applying (i) and (4.4), we have

$$
\begin{aligned}
P(X, \pi) & =P(A, \mathrm{id})\left(1+t^{2}+\cdots+t^{2(r-1)}\right) \\
& =\sum_{\alpha=0}^{d} \sum_{k=0}^{r-1}\left(b_{\alpha}(A)-b_{\alpha-2}(A)\right) t^{\alpha+2 k} s^{-\alpha+d}
\end{aligned}
$$

(iii) Let $i: Z \hookrightarrow A$ be a smooth subvariety of codimension $r$. Let $\pi: X \rightarrow A$ be a blow-up along $Z$. Using (4.3), we have

$$
\begin{aligned}
P(X, \pi)= & P(A, \mathrm{id})+P(Z, i)\left(t^{2}+t^{4}+\cdots+t^{2(r-1)}\right), \\
= & \sum_{\alpha=0}^{d}\left(b_{\alpha}(A)-b_{\alpha-2}(A)\right) t^{\alpha} s^{-\alpha+d} \\
& +\sum_{\alpha=0}^{d-r} \sum_{k=1}^{r-1}\left(b_{\alpha}(Z)-b_{\alpha-2}(Z)\right) t^{\alpha+2 k} s^{-\alpha+d-r} .
\end{aligned}
$$

Here we have used Lemma 4.5 next, which shows $P(Z, i)=P(Z$, id $)$. Lemma 4.5 will also be used in Lemma 5.5

Lemma 4.5. Let $u: A \rightarrow A^{\prime}$ be a finite morphism between projective varieties. Then the following diagram commutes,

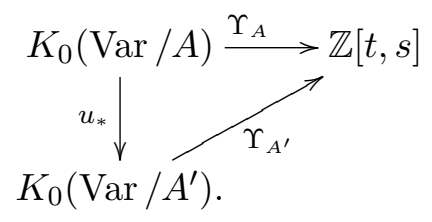

Here $u_{*}$ takes $\pi: X \rightarrow A$ to $u \circ \pi: X \rightarrow A \rightarrow A^{\prime}$. 
Proof. It is enough to check the commutativity for $[(X, \pi)] \in K_{0}(\operatorname{Var} / A)$, where $X$ is a smooth projective variety and $\pi: X \rightarrow A$ is a morphism. Let $H_{A^{\prime}}$ be an ample divisor on $A^{\prime}$, and set $H_{A}=u^{*} H_{A^{\prime}}$. Note that the divisor $H_{A}$ is also ample because $u$ is finite. Since the definition of $\Upsilon_{A}$ does not depend on a choice of an ample divisor on $A$, one may compute $\Upsilon_{A}(X, \pi)$ using the $e_{R^{-a c t i o n}}$ on $I H^{*}(X)$ given by $\pi^{*} H_{A} \wedge *$. Also one may compute $\Upsilon_{A^{\prime}}(X, u \circ \pi)$ by the $e_{R^{-a c t i o n}}$ given by $(u \circ \pi)^{*} H_{A^{\prime}} \wedge *$. Since $H_{A}=u^{*} H_{A^{\prime}}$, both $e_{R^{-a c t i o n s}}$ are same, hence $\Upsilon_{A}(X, \pi)=\Upsilon_{A^{\prime}}(X, u \circ \pi)$.

Remark 4.6. For $Q=\sum q^{a, b} t^{a} s^{b} \in \mathbb{Z}[t, s]$, let $m(Q)$ be the integer

$$
m(Q)=\max \left\{a+2 b \mid q^{a, b} \neq 0\right\} .
$$

Let $\pi: X \rightarrow A$ be a projective morphism with $X$ smooth and connected. Then it is clear that $m(Q)=2 \operatorname{dim} X$ for $Q=\Upsilon_{A}([(X, \pi)])$. In particular one can recover $\nu_{l}^{\alpha}$ from $t^{-m(Q) / 2} Q$. Using this and the motivic property of $\Upsilon_{A}$, one can easily show that for any quasi-projective variety $X$ with a morphism $\pi: X \rightarrow A$ and $Q=\Upsilon_{A}([(X, \pi)])$, the integer $m(Q)$ is even.

Based on Remark 4.6 we introduce the following notation, which will be used in Paragraph 4.4 .

Definition 4.7. We define the subset $\mathbb{Z}[t, s]^{\dagger} \subset \mathbb{Z}[t, s]$ to be the set of polynomials $Q$ such that $m(Q)$ is even. For $Q \in \mathbb{Z}[t, s]^{\dagger}$, we define the operation $Q^{b}$ by

$$
Q^{b}:=t^{-m(Q) / 2} Q \in \mathbb{Z}\left[t^{-1}, t, s\right]
$$

When $A=\operatorname{Spec} \mathbb{C}$, we write $K_{0}(\operatorname{Var} / A)$ as $K_{0}(\operatorname{Var} / \mathbb{C})$, and just write its elements as $[X] \in K_{0}(\operatorname{Var} / \mathbb{C})$ omitting the structure morphism $X \rightarrow$ Spec $\mathbb{C}$. Also we write $\Upsilon_{\text {Spec } \mathbb{C}}$ as $\Upsilon$ for simplicity.

Remark 4.8. If $A=\operatorname{Spec} \mathbb{C}$, all the Jordan cells have length one. Hence $\Upsilon(X) \in \mathbb{Z}[t]$ is nothing but the virtual Poincaré polynomial of $X$.

There is a ring structure on $K_{0}(\operatorname{Var} / \mathbb{C})$ defined by

$$
\left(\left[X_{1}\right],\left[X_{2}\right]\right) \longmapsto\left[X_{1} \times X_{2}\right]
$$

with unit $[$ Spec $\mathbb{C}]$. Also we have the following natural map,

$$
\Pi: K_{0}(\operatorname{Var} / \mathbb{C}) \times K_{0}(\operatorname{Var} / A) \longrightarrow K_{0}(\operatorname{Var} / A),
$$


which takes the pair $([T],[(X, \pi)])$ to

$$
T \times X \stackrel{\mathrm{pr}}{\longrightarrow} X \stackrel{\pi}{\longrightarrow} A,
$$

where pr is the projection to $X$. The operation $\Pi$ makes $K_{0}(\operatorname{Var} / A)$ a $K_{0}(\operatorname{Var} / \mathbb{C})$-algebra. We have the following lemma.

Lemma 4.9. The following diagram is commutative.

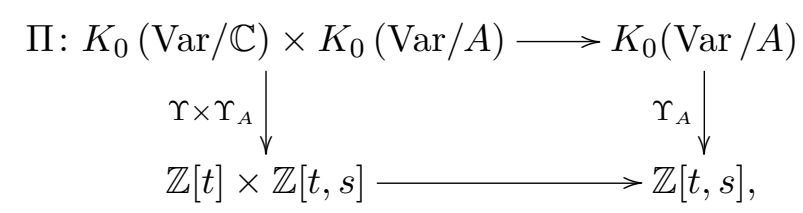

where the bottom arrow takes $\left(Q_{1}(t), Q_{2}(t, s)\right)$ to $Q_{1}(t) Q_{2}(t, s)$.

Proof. It is enough to check

$$
\Upsilon_{A}([T \times X, \pi \circ \mathrm{pr}])=\Upsilon(T) \cdot \Upsilon_{A}([(X, \pi)]),
$$

for smooth projective $T$ and $X$ with a morphism $\pi: X \rightarrow A$. We have the isomorphism as graded vector spaces,

$$
I H^{*}(T \times X) \cong I H^{*}(T) \otimes I H^{*}(X)
$$

Let us introduce the $e_{R}$-action on the right-hand side, by letting $e_{R}$ act on $I H^{*}(T)$ trivially. Then this isomorphism preserves the $e_{R}$-actions, which shows the equality (4.7) immediately.

Remark 4.10. More generally a motivic invariant is defined to be a ring homomorphism $\Upsilon: K(\operatorname{Var} / \mathbb{C}) \rightarrow \Lambda$ for some $\operatorname{ring} \Lambda$. Then a motivic invariant relative to $A$ is defined to be a map,

$$
K_{0}(\operatorname{Var} / A) \otimes_{K_{0}(\operatorname{Var} / \mathbb{C})} \Lambda \longrightarrow M
$$

for some $\Lambda$-module $M$. Lemma 4.9 implies that $\Upsilon_{A}$ is obtained in this way.

Remark 4.11. Let $\pi: X \rightarrow A$ be a morphism and $p: Z \rightarrow X$ be a Zariski locally trivial fibration with fiber $F$. Then we have $[(Z, \pi \circ p)]=\Pi([F]$, $[(X, \pi)])$, which yields the equality $\Upsilon_{A}([(Z, \pi \circ p)])=\Upsilon(F) \cdot \Upsilon_{A}([(X, \pi)])$ by Lemma 4.9 (cf. [24, lemma 4.2]). 


\subsection{Motivic invariants of Artin stacks}

Here we extend the invariant constructed in the previous paragraph to the invariant of Artin stacks over a projective variety $A$. The material of this paragraph is a slight generalization of Joyce's work [24]. In loc.cite, he works on the motivic invariants over $K_{0}(\operatorname{Var} / \mathbb{C})$ such as virtual Poincaré polynomials. For our purpose we have to extend the results in [24] to invariants over $K_{0}(\operatorname{Var} / A)$ such as $\Upsilon_{A}$. However the proofs are straightforward generalizations and we will leave some details to readers. Let $\mathcal{R}$ be an Artin stack of locally finite type over $\mathbb{C}$. Following Joyce [24], we introduce the Grothendieck group of Artin stacks over $\mathcal{R}$, denoted by $K_{0}(\mathrm{St} / \mathcal{R})$ in this paper. It was denoted by $\mathrm{SF}(\mathcal{R})$ in Joyce's papers [21-24].

Definition 4.12 [24, definition 3.1]. We define $K_{0}(\mathrm{St} / \mathcal{R})$ to be the $\mathbb{Q}$-vector space generated by equivalence classes of pairs $[(\mathcal{X}, \rho)]$, where $\rho: \mathcal{X} \rightarrow \mathcal{R}$ is a 1 -morphism of Artin stacks, $\mathcal{X}$ is of finite type over $\mathbb{C}$ with affine geometric stabilizers, such that for each closed substack $\mathcal{Y} \subset \mathcal{X}$, one has

$$
[(\mathcal{X}, \rho)]=[(\mathcal{Y}, \rho \mid \mathcal{Y})]+[(\mathcal{X} \backslash \mathcal{Y}, \rho \mid \mathcal{X} \backslash \mathcal{Y})]
$$

The following lemma is a generalization of [24, theorem 4.10]. Next we set $\Lambda=\mathbb{Q}(t, s)$.

Lemma 4.13. Let $A$ be a projective variety. Then $\Upsilon_{A}: K_{0}(\operatorname{Var} / A) \rightarrow$ $\mathbb{Z}[t, s]$ extends to the map,

$$
\Upsilon_{A}^{\prime}: K_{0}(\mathrm{St} / A) \longrightarrow \Lambda=\mathbb{Q}(t, s),
$$

such that for a 1-morphism $\rho: \mathcal{X} \rightarrow A$ with $\mathcal{X} \cong[X / G], X$ is a quasi-projective variety and $G$ a special algebraic $\mathbb{C}$-group (cf. [24, definition 2.1]), we have

$$
\Upsilon_{A}^{\prime}([(\mathcal{X}, \rho)])=\frac{\Upsilon_{A}([(X, \pi)])}{\Upsilon([G])} .
$$

Here $\pi$ is the composition, $\pi: X \rightarrow[X / G] \cong \mathcal{X} \stackrel{\rho}{\rightarrow} A$.

Proof. When $A=\operatorname{Spec} \mathbb{C}$, Lemma is proved in [24, theorem 4.10]. Also $\Upsilon([G])$ is non-zero in $\mathbb{Z}[t]$ by $[24$, lemma 4.7$]$, hence the right-hand side of (4.8) makes sense. We have to check that for $\rho: \mathcal{X} \rightarrow A$ with $\mathcal{X} 1$-isomorphic to $[X / G]$, the value $\Upsilon_{A}([(X, \pi)]) / \Upsilon([G])$ does not depend on a choice of $X$, $G$, and an isomorphism $\mathcal{X} \cong[X / G]$. This follows from Remark 4.11 and 
exactly the same proof of [24, proposition 4.8]. Finally as in the proof of [24, theorem 4.10], any Artin stack with affine geometric stabilizers $\mathcal{X}$ is stratified by global quotient stacks, and can define $\Upsilon_{A}^{\prime}([(\mathcal{X}, \rho)])$ by the formula $(4.8)$ and the linearity. Then the same proof of [24, theorem 4.10] shows that $\Upsilon_{A}^{\prime}([(\mathcal{X}, \rho)])$ does not depend on a choice of such a stratification.

We will use a $\Lambda$-module with more relations than $K_{0}(\mathrm{St} / \mathcal{R})$, denoted by $K_{0}(\mathrm{St} / \mathcal{R})$. It was denoted by $\operatorname{SF}(\mathcal{R}, \Upsilon, \Lambda)$ in $[24]$.

Definition 4.14 [24, definition 4.11]. We define $K_{0}($ St $/ \mathcal{R}) \Upsilon$ to be the $\Lambda$-module generated by equivalence classes of pairs $[(\mathcal{X}, \rho)]$, where $\rho: \mathcal{X} \rightarrow \mathcal{R}$ is a 1-morphism of Artin stacks, $\mathcal{X}$ is of finite type over $\mathbb{C}$ with affine geometric stabilizers, such that

(i) For each closed substack $\mathcal{Y} \subset \mathcal{X}$, one has

$$
[(\mathcal{X}, \rho)]=\left[\left(\mathcal{Y},\left.\rho\right|_{\mathcal{Y}}\right)\right]+\left[\left(\mathcal{X} \backslash \mathcal{Y},\left.\rho\right|_{\mathcal{X} \backslash \mathcal{Y})}\right)\right.
$$

(ii) Let $\mathcal{X}$ be a finite type Artin $\mathbb{C}$-stack with affine geometric stabilizers together with a 1 -morphism $\rho: \mathcal{X} \rightarrow \mathcal{R}$. Let $T$ be a quasi-projective variety, and pr: $T \times \mathcal{X} \rightarrow \mathcal{X}$ the projection. Then

$$
[(T \times \mathcal{X}, \rho \circ \mathrm{pr})]=\Upsilon([T])[(\mathcal{X}, \rho)]
$$

(iii) Let $\rho: \mathcal{X} \rightarrow \mathcal{R}$ be as before and $\mathcal{X} \cong[X / G]$ with $X$ quasi-projective, $G$ a special algebraic group acting on $X$. Then we have

$$
[(\mathcal{X}, \rho)]=\Upsilon([G])^{-1}[(X, \pi)]
$$

where $\pi$ is the composition $\pi: X \rightarrow[X / G] \cong \mathcal{X} \stackrel{\rho}{\rightarrow} \mathcal{R}$.

Remark 4.15. As in the proof of [24, theorem 4.10], any Artin stack of finite type is stratified by global quotient stacks. Then using (i), (iii) in Definition 4.14, one can show that $\Lambda$-module $K_{0}(\mathrm{St} / \mathcal{R})$ is spanned over $\Lambda$ by $[(X, \pi)]$, where $X$ is a variety and $\pi: X \rightarrow \mathcal{R}$ is a 1-morphism.

Now we descend the map $\Upsilon_{A}^{\prime}$ to $K_{0}(\mathrm{St} / A) \Upsilon$. 
Lemma 4.16. There is a $\Lambda$-module homomorphism $\bar{\Upsilon}_{A}: K_{0}(\mathrm{St} / A)_{\Upsilon} \rightarrow \Lambda$ such that the following diagram commutes,

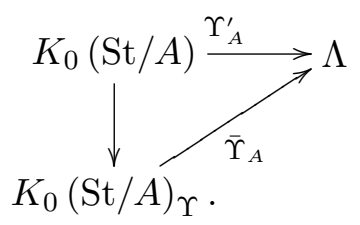

Here the left arrow is the natural quotient map.

Proof. For the relation (ii) of Definition 4.14, we have

$$
\Upsilon_{A}^{\prime}([(T \times \mathcal{X}, \rho \circ \mathrm{pr})])=\Upsilon(T) \cdot \Upsilon_{A}^{\prime}([\mathcal{X}, \rho])
$$

by Lemma 4.9 and the construction of $\Upsilon_{A}^{\prime}$ in Lemma 4.13. The compatibility with Definition 4.14 (iii) follows from (4.8).

Let $p: \mathcal{R}^{\prime} \rightarrow \mathcal{R}$ be a 1-morphism between Artin stacks of locally finite type. Then there is the notion of its push-forward,

$$
p_{*}: K_{0}\left(\mathrm{St} / \mathcal{R}^{\prime}\right) \longrightarrow K_{0}(\mathrm{St} / \mathcal{R})
$$

by taking a 1-morphism $\rho: \mathcal{X} \rightarrow \mathcal{R}^{\prime}$ to $p \circ \rho: \mathcal{X} \rightarrow \mathcal{R}$. Moreover if $p$ is of finite type, there is the notion of its pull-back,

$$
p^{*}: K_{0}(\mathrm{St} / R) \longrightarrow K_{0}\left(\mathrm{St} / \mathcal{R}^{\prime}\right)
$$

by taking a 1-morphism $\rho: \mathcal{X} \rightarrow \mathcal{R}$ to the fiber product $\mathcal{X} \times_{\mathcal{R}} \mathcal{R}^{\prime} \rightarrow \mathcal{R}^{\prime}$ (cf. [24, definition 3.4]). These operations descend to $\Lambda$-module homomorphisms between $K_{0}(\mathrm{St} / \mathcal{R}) \Upsilon$ and $K_{0}\left(\mathrm{St} / \mathcal{R}^{\prime}\right) \Upsilon(\mathrm{cf}$. [24, theorem 4.13]).

\subsection{Ringel-Hall product}

Let $\mathcal{M}$ be the moduli stack of $E \in \mathcal{D}_{X}$ satisfying (3.3), which we discussed in Section 3. Let $\mathcal{A} \subset \mathcal{D}_{X}$ be the heart of a bounded $t$-structure, and take 
$v \in N_{1}(X) \oplus \mathbb{Z}$. We consider the substacks

$$
\mathfrak{O b j}(\mathcal{A}) \subset \mathcal{M}, \quad \mathfrak{O b j}^{v}(\mathcal{A}) \subset \mathcal{M}
$$

the stack of objects in $\mathcal{A}$, the stack of objects in $\mathcal{A}$ of numerical type $v$, respectively. Suppose that $\mathfrak{O b j}(\mathcal{A})$ is an open substack of $\mathcal{M}$, hence in particular $\mathfrak{O} \mathfrak{b j}(\mathcal{A}), \mathfrak{O b j}^{v}(\mathcal{A})$ are Artin stacks of locally finite type. (This condition holds when $\mathcal{A}=\mathrm{Coh}_{\leq 1}(X)$.) Then there is an associative product $*$ on $K_{0}(\mathrm{St} / \mathfrak{O} \mathfrak{b j}(\mathcal{A}))$ and $K_{0}(\mathrm{St} / \mathfrak{O b j}(\mathcal{A}))_{\Upsilon}$, based on Ringel-Hall algebras [21]. Let $\mathfrak{E x}(\mathcal{A})$ be the stack of the exact sequences, $0 \rightarrow E_{1} \rightarrow E_{2} \rightarrow E_{3} \rightarrow 0$ in $\mathcal{A}$. We have the diagram,

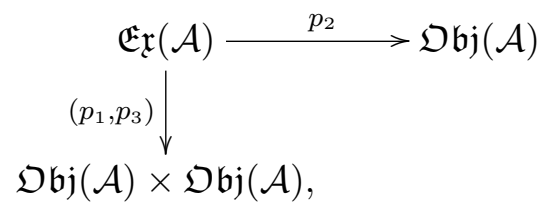

where $p_{i}$ takes $0 \rightarrow E_{1} \rightarrow E_{2} \rightarrow E_{3} \rightarrow 0$ to $E_{i}$. For elements $f_{i} \in K_{0}$ (St $/$ $\mathfrak{O b j}(\mathcal{A}))$ with $i=1,2$, one can define $f_{1} * f_{2}$ as

$$
f_{1} * f_{2}=p_{2 *}\left(p_{1} \times p_{3}\right)^{*}\left(f_{1} \times f_{2}\right) \in K_{0}(\operatorname{St} / \mathfrak{O b j}(\mathcal{A})),
$$

where $p_{2 *},\left(p_{1} \times p_{3}\right)^{*}$ are defined in (4.9), (4.10) respectively. Note that if $\left.f_{i} \in K_{0}(\mathrm{St} / \mathfrak{O} \mathfrak{b})^{v_{i}}(\mathcal{A})\right)$, the element $f_{1} * f_{2}$ is contained in $K_{0}\left(\mathrm{St} / \mathfrak{O} \mathfrak{b j}{ }^{v_{1}+v_{2}}\right.$ $(\mathcal{A})$ ). See $[21$, section 5] for the details.

Theorem 4.17 [21, theorem 5.2]. The operation * makes $K_{0}(\mathrm{St} / \mathfrak{O b j}$ $(\mathcal{A}))$ and $K_{0}(\operatorname{St} / \mathfrak{O b j}(\mathcal{A}))_{\Upsilon}$ associative algebras with unit $\left[\left(\operatorname{Spec} \mathbb{C}, \rho_{0}\right)\right]$, where $\rho_{0}: \operatorname{Spec} \mathbb{C} \rightarrow \mathfrak{O b j}(\mathcal{A})$ corresponds to the zero object.

In order to simplify the expositions in the following sections, we introduce Hall algebras of derived categories $(\mathcal{H}(X), *)$, introduced by Toën [43]. We emphasize that in our proof, we will not use the algebra $(\mathcal{H}(X), *)$ essentially. The algebra $(\mathcal{H}(X), *)$ contains $\left(K_{0}(\operatorname{St} / \mathfrak{O b j}(\mathcal{A})), *\right)$ as a subalgebra, and all the computations in the proof will be made in the latter algebra for suitable hearts of bounded $t$-structures $\mathcal{A} \subset \mathcal{D}_{X}$. As we do not need its actual definition, we only give its rough explanation and properties. Let $\widetilde{\mathcal{D}}$ be a dg-category of finite type (cf. [45, definition 2.4]) whose homotopy category is $D(X)$. Then Toën and Vaquié [45, theorem 0.1$]$ showed that the stack of objects in $\widetilde{\mathcal{D}}$, which they denote $\operatorname{Perf}(X)$ in [45, definition 3.28], is an $\infty$-stack of locally geometric and locally of finite presentation. Then 
$\mathcal{H}(X)$ is defined by the $\infty$-stack version of our notion of stack functions over $\operatorname{Per} f(X)$ (in [43, paragraph 3.3], it is denoted by $\mathcal{H}_{a b s}(\widetilde{\mathcal{D}})$,) and the *-product is defined in the similar way of (4.12). Furthermore by [45, corol-

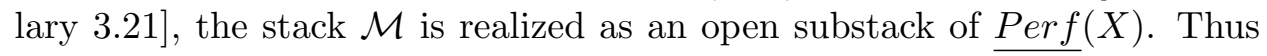
if there is a 1-morphism,

$$
\rho: \mathcal{X} \longrightarrow \mathcal{M}
$$

with $\mathcal{X}$ an Artin stack of finite type, it defines an element $[(\mathcal{X}, \rho)] \in \mathcal{H}(X)$. In particular for the heart of a bounded $t$-structure $\mathcal{A} \subset \mathcal{D}_{X}$ with $\mathfrak{O b j}(\mathcal{A}) \subset$ $\mathcal{M}$ open, the algebra $\left(K_{0}(\operatorname{St} / \mathfrak{O b j}(\mathcal{A})), *\right)$ is realized as a subalgebra of $\mathcal{H}(X)$ (see [45, 46 - paragraph 3.3] for details).

\subsection{Counting invariants of moduli stacks}

Next we assume $X$ is a Calabi-Yau threefold, and use the space of stability conditions $\operatorname{Stab}(X)$ and the open subset $U_{X}$ in Section 3. Let us take $v \in$ $N_{1}(X) \oplus \mathbb{Z}, \sigma \in \bar{U}_{X}$ and $\phi \in \mathbb{R}$. We consider the substack

$$
i: \mathcal{M}^{(v, \phi)}(\sigma) \hookrightarrow \mathcal{M}
$$

which is the moduli stack of $E \in \mathcal{P}(\phi)$ of numerical type $v$. By Proposition 3.9, $\mathcal{M}^{(v, \phi)}(\sigma)$ is an Artin stack of finite type. Also for an interval $I \subset \mathbb{R}$, let $C_{\sigma}(I) \subset N_{1}(X) \oplus \mathbb{Z}$ be the image of the map,

$$
\left(\operatorname{ch}_{2}, \operatorname{ch}_{3}\right): \mathcal{P}(I) \backslash\{0\} \longrightarrow N_{1}(X) \oplus \mathbb{Z}
$$

Definition 4.18. For $\sigma \in \bar{U}_{X}$, we define $\delta^{(v, \phi)}(\sigma) \in \mathcal{H}(X)$ to be

$$
\delta^{(v, \phi)}(\sigma)=\left[\left(\mathcal{M}^{(v, \phi)}(\sigma), i\right)\right] \in \mathcal{H}(X)
$$

Also define $\epsilon^{(v, \phi)}(\sigma) \in \mathcal{H}(X)$ as follows,

$$
\epsilon^{(v, \phi)}(\sigma)=\sum_{v_{1}+\cdots+v_{n}=v} \frac{(-1)^{n-1}}{n} \delta^{\left(v_{1}, \phi\right)}(\sigma) * \cdots * \delta^{\left(v_{n}, \phi\right)}(\sigma),
$$

where $v_{i} \in C_{\sigma}(\phi)$.

We have to check the following, whose proof will be given in Section 6 .

Lemma 4.19. The sum (4.13) is a finite sum. 
Remark 4.20. Suppose that for $\phi \in \mathbb{R}$, there is the heart of a $t$-structure $\mathcal{A} \subset \mathcal{D}_{X}$ such that $\mathfrak{O} \mathfrak{b j}(\mathcal{A}) \subset \mathcal{M}$ is open and $\mathcal{P}(\phi) \subset \mathcal{A}$. Then $\delta^{(v, \phi)}(\sigma)$ is contained in the subalgebra $K_{0}(\operatorname{St} / \mathfrak{O b j}(\mathcal{A}))$ and $\epsilon^{(v, \phi)}(\sigma)$ coincides with the one defined in the algebra $K_{0}(\mathrm{St} / \mathfrak{O b j}(\mathcal{A}))$ as in [22, definition 8.1].

Remark 4.21. Suppose any $\sigma$-semistable object of numerical type $v$ is stable. Then we must have $\epsilon^{(v, \phi)}(\sigma)=\delta^{(v, \phi)}(\sigma)$.

For $v=(\beta, k) \in N_{1}(X) \oplus \mathbb{Z}$, let us consider the following open substacks of $\mathcal{M}$,

$$
\mathfrak{C o h}(X):=\mathfrak{O b j}\left(\mathrm{Coh}_{\leq 1}(X)\right), \quad \mathfrak{C o h}^{v}(X):=\mathfrak{O b j}^{v}\left(\operatorname{Coh}_{\leq 1}(X)\right)
$$

By Remark 4.20, for $0<\phi \leq 1$ and $\sigma \in U_{X}$, we have

$$
\epsilon^{(v, \phi)}(\sigma) \in K_{0}\left(\mathrm{St} / \mathfrak{C o h}^{v}(X)\right)
$$

Using the same argument as in [37, chapter 5, section 4], we have the following 1-morphism,

$$
\pi: \mathfrak{C o h}^{v}(X) \ni E \longmapsto s(E) \in \operatorname{Chow}_{\beta}(X) .
$$

Now we define the element $P(v, \sigma) \in \Lambda$.

Definition 4.22. For $v \in N_{1}(X) \oplus \mathbb{Z}$ and $\sigma \in U_{X}$ as before, we define $P(v, \sigma) \in \Lambda$ as follows.

- If $v \in C_{\sigma}(\phi)$ with $0<\phi \leq 1$, we define

$$
P(v, \sigma):=(\mathbb{L}-1) \Upsilon_{A}^{\prime}\left(\pi_{*} \epsilon^{(v, \phi)}(\sigma)\right),
$$

where $\mathbb{L}=\Upsilon\left(\mathbb{A}^{1}\right)$ and $A=\operatorname{Chow}_{\beta}(X)$. Note that $\pi_{*} \epsilon^{(v, \phi)}(\sigma) \in K_{0}$ (St $/ A)$ makes sense by (4.14).

- If $v \in C_{\sigma}(\phi)$ with $1<\phi \leq 2$, we define

$$
P(v, \sigma):=P(-v, \sigma)
$$

Note that in this case $-v \in C_{\sigma}(\phi-1)$, hence the right-hand side makes sense.

- Otherwise we define $P(v, \sigma)=0$. 
Remark 4.23. The definition of $P(v, \sigma)$ for $v \in C_{\sigma}(\phi)$ with $1<\phi \leq 2$ is motivated by the following observation. Let $\mathfrak{C o h}^{v}(X)[1]$ be the stack of objects $E \in \operatorname{Coh}_{\leq 1}(X)[1]$ of numerical type $v$. Then we have

$$
\epsilon^{(v, \phi)}(\sigma) \in K_{0}\left(\mathrm{St} / \mathfrak{C o h}^{v}(X)[1]\right) .
$$

Also we have the 1-morphism,

$$
\pi^{\prime}: \mathfrak{C o h}^{v}(X)[1] \ni E \longmapsto-s(E) \in A^{\prime}:=\mathrm{Chow}_{-\beta}(X) .
$$

Hence it is reasonable to define

$$
P(v, \sigma)=(\mathbb{L}-1) \Upsilon_{A^{\prime}}^{\prime}\left(\pi_{*}^{\prime} \epsilon^{(v, \phi)}(\sigma)\right)
$$

However we can easily see that the right-hand side of (4.15) is equal to $P(-v, \sigma)$, hence we define $P(v, \sigma)$ as in Definition 4.22 to reduce the exposition.

We have the following proposition. (Recall that we have defined $\mathbb{Z}[t, s]^{\dagger}$ and $Q^{b}$ for $Q \in \mathbb{Z}[t, s]^{\dagger}$ in Definition 4.7.)

Proposition 4.24. If $\sigma=\sigma_{(0, \omega)}$ with $\omega$ ample and $v=(\beta, 1)$, we have $P(v, \sigma) \in \mathbb{Z}[t, s]^{\dagger}$.

Proof. We may assume $v \in C_{\sigma}(\phi)$ for $0<\phi \leq 1$. If $\sigma=\sigma_{(0, \omega)}$ and $v=(\beta, 1)$, any semistable object in $\sigma$ of type $v$ is stable. In fact suppose there is a $\sigma$-semistable object $E \in \mathrm{Coh}_{\leq 1}(X)$ which is not stable. Then there is an exact sequence $0 \rightarrow E_{1} \rightarrow E \rightarrow E_{2} \rightarrow 0$ in $\operatorname{Coh}_{\leq 1}(X)$ such that

$$
\arg Z_{(0, \omega)}(E)=\arg Z_{(0, \omega)}\left(E_{1}\right)=\arg Z_{(0, \omega)}\left(E_{2}\right)
$$

Because $\operatorname{ch}_{3}(E)=1$, we have $\operatorname{Re} Z_{(0, \omega)}(E)<0$, hence (4.16) implies $\operatorname{Re} Z_{(0, \omega)}$ $\left(E_{i}\right)<0$ for $i=1,2$. Since $-\operatorname{ch}_{3}\left(E_{i}\right)=\operatorname{Re} Z_{(0, \omega)}\left(E_{i}\right)$, this contradicts to $1=\operatorname{ch}_{3}(E)=\operatorname{ch}_{3}\left(E_{1}\right)+\operatorname{ch}_{3}\left(E_{2}\right)$.

By Remark 4.21 we have

$$
\epsilon^{(v, \phi)}(\sigma)=\delta^{(v, \phi)}(\sigma)=\left[\left(\left[M^{v} / \mathbb{G}_{m}\right], \rho\right)\right]
$$

for some projective variety $M^{v}, \mathbb{G}_{m}$ acting on $M^{v}$ trivially and $\rho$ is a 1-morphism $\left[M^{v} / \mathbb{G}_{m}\right] \rightarrow \mathfrak{C o h}(X)$. (The factor $\mathbb{G}_{m}$ comes from the stabilizers $\operatorname{Aut}(E) \cong \mathbb{G}_{m}$ for stable objects $E$.) In fact $M^{v}$ is the moduli space of $\omega$-Gieseker stable sheaves of Chern character $v$. Thus by Remark 4.6 and noting $\mathbb{L}-1=\Upsilon\left(\mathbb{G}_{m}\right)$, we have $P(v, \sigma) \in \mathbb{Z}[t, s]^{\dagger}$. 
Now we define the notion of motivic Gopakumar-Vafa invariant.

Definition 4.25. For $\sigma=\sigma_{(0, \omega)} \in U_{X}$ and $v=(\beta, 1)$, write $P(v, \sigma)^{b}$ as

$$
P(v, \sigma)^{b}=\sum_{\alpha, l} \nu_{l}^{\alpha}(\beta) t^{\alpha} s^{l-1} .
$$

By Proposition 4.24, it is possible to define $P(v, \sigma)^{b}$. Then define the motivic Gopakumar-Vafa invariant $n_{g}^{\beta}(X)$ as follows:

$$
\begin{aligned}
n_{g}^{\beta}(X)= & \sum_{\alpha+l \geq 1}(-1)^{\alpha+g} l \nu_{l}^{\alpha}(\beta) \\
& \times\left\{\left(\begin{array}{c}
\alpha+l+g \\
2 g+1
\end{array}\right)-\left(\begin{array}{c}
\alpha+l+g-2 \\
2 g+1
\end{array}\right)\right\} .
\end{aligned}
$$

Remark 4.26. The motivation of (4.17) in Definition 4.25 comes from Proposition 2.5. Obviously the invariant $n_{g}^{\beta}(X)$ coincides with $\tilde{n}_{g}^{\beta}$ if $\beta$ is represented by an effective one cycle, and the moduli space $M^{\beta}$ in Paragraph 2.3 is smooth.

Remark 4.27. Still the definition of $n_{g}^{\beta}$ does not involve virtual classes, so $n_{g}^{\beta}$ is unlikely to be deformation invariant. On the other hand, our construction of BPS-count has a possibility to involve virtual classes using Behrend's constructible functions [3]. As in the proof of Proposition 4.24, $P(v, \sigma)$ in Definition 4.25 is equal to $\Upsilon_{A}\left(\left[\left(M^{v}, \rho\right)\right]\right)$ for some projective variety over $A$, $\rho: M^{v} \rightarrow A$. Then using Behrend's constructible function $\nu: M^{v} \rightarrow \mathbb{Z}$, one might try to define $P^{\prime}(v, \sigma)$ something like

$$
P^{\prime}(v, \sigma)=\sum_{n} \pm n \Upsilon_{A}\left(\left[\nu^{-1}(n),\left.\rho\right|_{\nu^{-1}(n)}\right]\right)
$$

and construct $n_{g}^{\beta^{\prime}}$ by the same way as in Definition 4.25. In this paper we stick to Definition 4.25 in order to show birational invariance, though it seems interesting to pursue this construction.

In the next paragraph, we will show that $n_{g}^{\beta}(X)$ does not depend on a choice of $\omega$. 


\subsection{Local transformation formula of the counting invariants}

The aim of this paragraph is to give the transformation formula of $\epsilon^{(v, \phi)}(\sigma)$ under small deformations of $\sigma$. Again we assume $X$ is a Calabi-Yau threefold. Let us fix the following data:

$$
v \in N_{1}(X) \oplus \mathbb{Z}, \quad \phi \in \mathbb{R}, \quad \sigma=(Z, \mathcal{P}) \in \bar{U}_{X}
$$

Furthermore we fix an open neighborhood $\sigma \in \mathcal{U}$ in $\operatorname{Stab}(X)$ such that $\overline{\mathcal{U}}$ is compact. We set $\mathcal{S} \subset \mathcal{D}_{X}$ to be the set of objects,

$$
\begin{gathered}
\mathcal{S}:=\left\{E \in \mathcal{D}_{X} \mid E \text { is semistable in some } \sigma^{\prime}=\left(Z^{\prime}, \mathcal{P}^{\prime}\right) \in \overline{\mathcal{U}}\right. \text { with } \\
\left.\left|Z^{\prime}(E)\right| \leq\left|Z^{\prime}(v)\right|\right\}
\end{gathered}
$$

Then $\mathcal{S}$ has bounded mass, hence there is a wall and chamber structure on $\overline{\mathcal{U}}$ as in Proposition 3.7. Let $\mathcal{C} \subset \overline{\mathcal{U}}$ be a chamber with $\sigma \in \overline{\mathcal{C}}$. By the definition of the topology on $\operatorname{Stab}(X)$ (cf. [7, Section 6]), we can take $\tau=(W, \mathcal{Q}) \in$ $U_{X} \cap \mathcal{C}$ and $0<\varepsilon<1 / 6$ such that

$$
\mathcal{P}(\phi) \subset \mathcal{Q}((\phi-\varepsilon, \phi+\varepsilon)), \quad \mathcal{Q}(\phi) \subset \mathcal{P}((\phi-\varepsilon, \phi+\varepsilon))
$$

for any $\phi \in \mathbb{R}$. Furthermore we can take $W$ to be defined over $\mathbb{Q}$. Note that for any $v \in C_{\tau}((\phi-\varepsilon, \phi+\varepsilon))$, there are uniquely determined $\phi(v), \phi^{\prime}(v) \in$ $\left(\phi-\frac{1}{2}, \phi+\frac{1}{2}\right)$ such that

$$
W(v) \in \mathbb{R}_{>0} \exp (\pi i \phi(v)), \quad Z(v) \in \mathbb{R}_{>0} \exp \left(\pi i \phi^{\prime}(v)\right)
$$

by our choice of $\varepsilon$. Recall the definition of $\mathbb{H} \subset \mathbb{C}$ in (3.1).

Proposition 4.28. Let $\sigma, \tau$ and $\varepsilon>0$ be as before. There is a unique sequence of functions $u_{n}: \mathbb{H}^{2 n} \rightarrow \mathbb{Q}$ such that we have the following in $\mathcal{H}(X)$,

$$
\begin{aligned}
\epsilon^{(v, \phi)}(\sigma)= & \sum_{v_{1}+\cdots+v_{n}=v} u_{n}\left(z_{1}, \ldots, z_{n}, w_{1}, \ldots, w_{n}\right) \\
& \times \epsilon^{\left(v_{1}, \phi_{1}\right)}(\tau) * \cdots * \epsilon^{\left(v_{n}, \phi_{n}\right)}(\tau) \\
= & \epsilon^{(v, \phi)}(\tau)+\left[\text { multiple commutators of } \epsilon^{\left(v_{i}, \phi_{i}\right)}(\tau)\right]
\end{aligned}
$$

where $v_{i} \in C_{\tau}((\phi-\varepsilon, \phi+\varepsilon)), \phi_{i}=\phi\left(v_{i}\right)$ and

$$
z_{i}=\exp \left(-\pi i\left(\phi-\frac{1}{2}\right)\right) Z\left(v_{i}\right), \quad w_{i}=\exp \left(-\pi i\left(\phi-\frac{1}{2}\right)\right) W\left(v_{i}\right)
$$


Here (4.19) is a finite sum, and $[\cdots]$ in (4.20) is a finite $\mathbb{Q}$-linear combination of multiple commutators of $\epsilon^{\left(v_{i}, \phi_{i}\right)}(\tau)$.

Proof. This is an application of the arguments in [23, theorem 5.2] to Bridgeland's stability conditions, and the proof is same as in [41, equation (68)]. Note that (4.18) implies $z_{i}, w_{i} \in \mathbb{H}$, thus (4.19) makes sense. By [41, proposition 3.18], there is $\psi \in \mathbb{R}$ such that

$$
\psi-1<\phi-\varepsilon<\phi+\varepsilon<\psi
$$

and $\mathfrak{O b j}\left(\mathcal{A}_{\psi}\right) \subset \mathcal{M}$ is open for $\mathcal{A}_{\psi}=\mathcal{Q}((\psi-1, \psi])$. Since all the terms in (4.19) are contained in $K_{0}\left(\mathrm{St} / \mathfrak{O} \mathfrak{b j}\left(\mathcal{A}_{\psi}\right)\right)$, it is enough to show (4.19) in $K_{0}\left(\operatorname{St} / \mathfrak{O} \mathfrak{b j}\left(\mathcal{A}_{\psi}\right)\right)$. Then the straightforward adaptation of the arguments in [41, proposition 5.23] which deduces [41, equation (68)] gives the desired equality. (Note that in loc.cite, we worked over an algebra $A\left(\mathcal{A}_{\psi}, \Lambda, \chi\right)$, not over $K_{0}\left(\mathrm{St} / \mathfrak{O} \mathfrak{b j}\left(\mathcal{A}_{\psi}\right)\right)$.) However readers can find that the same argument is applied. Moreover in loc.cite, the coefficients are given in the form $U\left(\left\{v_{i}\right\}_{1 \leq i \leq n}, \tau, \sigma\right)$. These are obviously rephrased in terms of the functions $u_{n}: \mathbb{H}^{2 n} \rightarrow \mathbb{Q}$, as in [25, paragraph 3.1]. Finally the formula (4.20) follows from [23, theorem 5.2].

Remark 4.29. Suppose that there is the heart of a $t$-structure $\mathcal{A} \subset \mathcal{D}_{X}$ with $\mathfrak{O b j}(\mathcal{A}) \subset \mathcal{M}$ open, and all the terms $\epsilon^{(v, \phi)}(\sigma), \epsilon^{\left(v_{i}, \phi_{i}\right)}(\tau)$ in (4.19) are contained in $K_{0}(\mathrm{St} / \mathfrak{O b j}(\mathcal{A}))$. Then (4.19), (4.20) hold in the algebra $K_{0}(\operatorname{St} / \mathfrak{O b j}(\mathcal{A}))$.

The explicit formula of $u_{n}$ (cf. [23, definition 4.4]) is complicated and we do not need this. Now we show the following proposition.

Proposition 4.30. For any $v=(\beta, k) \in N_{1}(X) \oplus \mathbb{Z}$, the element $P(v, \sigma) \in$ $\Lambda$ does not depend on a choice of $\sigma \in U_{X}$.

Proof. For $\sigma \in U_{X}$, let us take $\tau \in U_{X}$ and $\varepsilon>0$ as in Proposition 4.28. It is enough to show $P(v, \sigma)=P(v, \tau)$ in this situation. First assume $v \notin C_{\sigma}(\phi)$ for any $\phi \in \mathbb{R}$, thus $P(v, \sigma)=0$. If $P(v, \tau) \neq 0$, there is some $\tau$-semistable object $E \in \mathcal{D}_{X}$ of numerical type $v$. Because $\tau$ is contained in a chamber, $E$ must be also semistable in $\sigma$, which is a contradiction. (See the comment in [7] after [7, proposition 8.1].) Hence $P(v, \tau)=0$ follows. 
Next suppose $v \in C_{\sigma}(\phi)$ for some $\phi$. We may assume $0<\phi \leq 1$. If $\phi=1$, then $\beta=0$ and $v \in C_{\tau}(1)$. Since we have

$$
\mathcal{P}(1)=\mathcal{Q}(1)=\{\text { zero-dimensional sheaves }\}
$$

it follows that $\delta^{\left(v_{i}, 1\right)}(\sigma)=\delta^{\left(v_{i}, 1\right)}(\tau)$ for any $v_{i} \in C_{\sigma}(1)=C_{\tau}(1)$. Hence $\epsilon^{(v, 1)}$ $(\sigma)=\epsilon^{(v, 1)}(\tau)$ and $P(v, \sigma)=P(v, \tau)$ follows.

Finally suppose $0<\phi<1$. We can take $\varepsilon>0$ sufficiently small such that $0<\phi-\varepsilon<\phi+\varepsilon<1$. Then all the terms $\epsilon^{\left(v_{i}, \phi_{i}\right)}(\tau)$ in (4.19) are contained in $K_{0}(\mathrm{St} / \mathfrak{C o h}(X))$, and (4.20) holds in $K_{0}(\mathrm{St} / \mathfrak{C o h}(X))$. Then applying Lemma 4.16 and Remark 4.15, it is enough to show the following: for two varieties $U_{1}, U_{2}$ with 1 -morphisms $\rho_{i}: U_{i} \rightarrow \mathfrak{C o h}^{v_{i}}(X)$, where $v_{i} \in C_{\tau}((\phi-$ $\varepsilon, \phi+\varepsilon))$ with $v_{1}+v_{2}=v$, we have

$$
\Upsilon_{A}^{\prime}\left(\pi_{*}\left[f_{1}, f_{2}\right]\right)=0, \quad f_{i}=\left[\left(U_{i}, \rho_{i}\right)\right] \in K_{0}(\mathrm{St} / \mathfrak{C o h}(X))
$$

where $A=\operatorname{Chow}_{\beta}(X)$. Note that if there is an exact sequence $0 \rightarrow E_{1} \rightarrow$ $E \rightarrow E_{2} \rightarrow 0$ in $\operatorname{Coh}_{\leq 1}(X)$, we have $s(E)=s\left(E_{1} \oplus E_{2}\right)$. Using this and Lemma 4.31 next, we can conclude (4.21) holds.

Lemma 4.31. Let $\mathcal{A} \subset \mathcal{D}_{X}$ be the heart of a t-structure with $\mathfrak{O b j}(\mathcal{A}) \subset \mathcal{M}$ open. For $v \in N_{1}(X) \oplus \mathbb{Z}$, assume that there is a 1-morphism $\pi: \mathfrak{O b j}^{v}(\mathcal{A}) \rightarrow$ $A$, where $A$ is a projective variety, which satisfies,

$$
\begin{aligned}
\pi([E])= & \pi\left(\left[E_{1} \oplus E_{2}\right]\right), \quad \text { for any exact sequence } \\
& 0 \rightarrow E_{1} \rightarrow E \rightarrow E_{2} \rightarrow 0 \text { in } \mathcal{A}
\end{aligned}
$$

where $E \in \mathcal{A}$ is of numerical type $v$. Then we have

$$
\Upsilon_{A}^{\prime}\left(\pi_{*}\left[f_{1}, f_{2}\right]\right)=0, \quad f_{i}=\left[\left(U_{i}, \rho_{i}\right)\right] \in K_{0}(\operatorname{St} / \mathfrak{O b j}(\mathcal{A})),
$$

where $U_{1}, U_{2}$ are quasi-projective varieties.

Proof. For $\mathbb{C}$-valued points $p_{i} \in U_{i}$, let $E\left(p_{i}\right) \in \mathcal{A}$ be the objects corresponding to $\rho_{i}\left(p_{i}\right)$. Let us decompose $U_{1} \times U_{2}$ into finite locally closed pieces,

$$
U_{1} \times U_{2}=\coprod_{k} W_{k},
$$

such that the dimensions of $\operatorname{Ext}^{j}\left(E\left(p_{1}\right), E\left(p_{2}\right)\right), \operatorname{Ext}^{j}\left(E\left(p_{2}\right), E\left(p_{1}\right)\right)$ are constant on each $W_{k}$ for $j=0,1$. Furthermore we may assume that the 
bundles

$\bigcup_{\left(p_{1}, p_{1}\right) \in W_{k}} \operatorname{Ext}^{j}\left(E\left(p_{2}\right), E\left(p_{1}\right)\right) \rightarrow W_{k}, \quad \bigcup_{\left(p_{1}, p_{2}\right) \in W_{k}} \operatorname{Ext}^{j}\left(E\left(p_{1}\right), E\left(p_{2}\right)\right) \longrightarrow W_{k}$

are trivial bundles with fibers $V_{k}^{j}, \bar{V}_{k}^{j}$ for $j=0,1$ respectively. Let us consider the diagram (4.11) which defines $*$-product on $K_{0}(\operatorname{St} / \mathfrak{O b j}(\mathcal{A}))$. Then the set of $\mathbb{C}$-valued points of the following stack,

$$
W_{k} \times \mathfrak{O b j}(\mathcal{A}) \times \mathfrak{O} \mathfrak{b j}(\mathcal{A}) \mathfrak{E} \mathfrak{x}(\mathcal{A})
$$

is identified with the $\mathbb{C}$-valued points of $W_{k} \times V_{k}^{1}$. Let $0 \rightarrow E\left(p_{1}\right) \rightarrow E \rightarrow$ $E\left(p_{2}\right) \rightarrow 0$ be an exact sequence in $\mathcal{A}$ which represents a $\mathbb{C}$-valued point of (4.24). Then the stabilizers at this point in (4.24) is identified with the fiber at (id, id) of the following morphism,

$$
\operatorname{Aut}\left(0 \longrightarrow E\left(p_{1}\right) \longrightarrow E \longrightarrow E\left(p_{2}\right) \longrightarrow 0\right) \longrightarrow \operatorname{Aut}\left(E\left(p_{1}\right)\right) \times \operatorname{Aut}\left(E\left(p_{2}\right)\right)
$$

which is isomorphic to $V_{k}^{0}=\operatorname{Hom}\left(E\left(p_{2}\right), E\left(p_{1}\right)\right)$. Hence we have

$$
\left(p_{1}, p_{3}\right)^{*}\left\{\left.\left(f_{1}, f_{2}\right)\right|_{W_{k}}\right\}=\left[W_{k} \times V_{k}^{1} / V_{k}^{0}\right]
$$

where $V_{k}^{0}$ acts on $W_{k} \times V_{k}^{1}$ trivially. Therefore we can write $f_{1} * f_{2}$ in the following form,

$$
f_{1} * f_{2}=\sum_{k}\left[\left(\left[W_{k} \times V_{k}^{1} / V_{k}^{0}\right], \rho_{k}^{\prime}\right)\right]
$$

for some 1-morphism $\rho_{k}^{\prime}:\left[W_{k} \times V_{k}^{1} / V_{k}^{0}\right] \rightarrow \mathfrak{O b j}^{v}(\mathcal{A})$. (Also see [23, theorem 5.18].) Let us consider the composition,

$$
W_{k} \times V_{k}^{1} \longrightarrow\left[W_{k} \times V_{k}^{1} / V_{k}^{0}\right] \stackrel{\rho_{k}^{\prime}}{\longrightarrow} \mathfrak{O b j}^{v}(\mathcal{A}) \stackrel{\pi}{\longrightarrow} A
$$

By the assumption (4.22), the earlier morphism is nothing but the following map,

$$
W_{k} \times V_{k}^{1} \ni\left(p_{1}, p_{2}, v\right) \longmapsto \pi\left(\left[E\left(p_{1}\right) \oplus E\left(p_{2}\right)\right]\right)
$$

Hence the morphism (4.25) descends to the morphism, $\rho_{k}^{\dagger}: W_{k} \rightarrow A$, which takes $\left(p_{1}, p_{2}\right)$ to $\pi\left(\left[E\left(p_{1}\right) \oplus E\left(p_{2}\right)\right]\right)$. Therefore by Lemma 4.9 and 
using (4.8), we have

$$
\Upsilon_{A}^{\prime}\left(\pi_{*}\left(f_{1} * f_{2}\right)\right)=\sum_{k} \mathbb{L}^{\operatorname{dim} V_{k}^{1}-\operatorname{dim} V_{k}^{0}} \Upsilon_{A}\left(\left[\left(W_{k}, \rho_{k}^{\dagger}\right)\right]\right)
$$

(Recall that we have defined $\mathbb{L}=\Upsilon\left(\mathbb{A}^{1}\right)$ in Definition 4.22.) Arguing as in the same way for $\Upsilon_{A}^{\prime}\left(\pi_{*}\left(f_{2} * f_{1}\right)\right)$ and taking their difference, we obtain

$$
\Upsilon_{A}^{\prime}\left(\pi_{*}\left[f_{1}, f_{2}\right]\right)=\sum_{k}\left(\mathbb{L}^{\operatorname{dim} V_{k}^{1}-\operatorname{dim} V_{k}^{0}}-\mathbb{L}^{\operatorname{dim} \bar{V}_{k}^{1}-\operatorname{dim} \bar{V}_{k}^{0}}\right) \Upsilon_{A}\left(\left[\left(W_{k}, \rho_{k}^{\dagger}\right)\right]\right)
$$

Then Sublemma 4.32 next shows $\operatorname{dim} V_{k}^{1}-\operatorname{dim} V_{k}^{0}=\operatorname{dim} \bar{V}_{k}^{1}-\operatorname{dim} \bar{V}_{k}^{0}$, hence (4.21) follows.

We have used the following sublemma.

Sublemma 4.32. Let $\mathcal{A} \subset \mathcal{D}_{X}$ be the heart of a t-structure and take $E, F \in$ $\mathcal{A}$. Then one has

$$
\operatorname{dim} \operatorname{Ext}^{1}(E, F)-\operatorname{dim} \operatorname{Hom}(E, F)=\operatorname{dim} \operatorname{Ext}^{1}(F, E)-\operatorname{dim} \operatorname{Hom}(F, E) .
$$

Proof. Since $X$ is a Calabi-Yau threefold, we have

$$
\begin{aligned}
& \chi(E, F):=\sum_{k}(-1)^{k} \operatorname{dim} \operatorname{Ext}^{k}(E, F) \\
& =-\operatorname{dim} \operatorname{Ext}^{1}(E, F)+\operatorname{dim} \operatorname{Hom}(E, F) \\
& +\operatorname{dim} \operatorname{Ext}^{1}(F, E)-\operatorname{dim} \operatorname{Hom}(F, E) \text {, }
\end{aligned}
$$

by Serre duality. On the other hand Riemann-Roch implies $\chi(E, F)=0$ because $\operatorname{ch}_{i}(E)=\operatorname{ch}_{i}(F)=0$ for $i=0,1$.

Combined with Proposition 4.24, we have the following.

Corollary 4.33. For any $\sigma \in U_{X}$ and $v=(\beta, 1)$, we have $P(v, \sigma)=P(v$, $\left.\sigma_{(0, \omega)}\right) \in \mathbb{Z}[t, s]^{\dagger}$, and $n_{g}^{\beta}(X)$ does not depend on a choice of $\omega$.

\section{Birational invariance of the counting invariants}

Now we state our main theorem.

Theorem 5.1. Let $\phi: W \rightarrow X$ be a birational map between smooth projective Calabi-Yau threefolds. Then for $\beta \in N_{1}(W)$, one has

$$
n_{g}^{\beta}(W)=n_{g}^{\phi_{*} \beta}(X)
$$


The strategy is as follows. First we enlarge the definition of $P(v, \sigma) \in$ $\Lambda$ for some boundary points $\sigma \in \bar{U}_{X}$, and show $P(v, \sigma)=P(v, \tau)$ for $\tau \in$ $U_{X}$. Next we compare $P(v, \sigma)$ with $P\left(v^{\prime}, \sigma^{\prime}\right)$ defined for $\sigma^{\prime} \in \bar{U}_{W}$, using the derived equivalence [8], $\Phi: \mathcal{D}_{W} \rightarrow \mathcal{D}_{X}$.

\subsection{Perverse $t$-structures on $\mathcal{D}_{X}$}

Before giving the proof of Theorem 5.1, we investigate some boundary points in $\bar{U}_{X}$. We assume there is a diagram of birational maps,

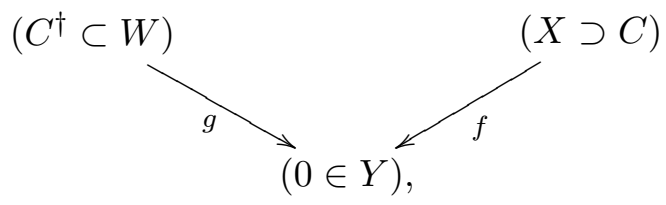

where $C$ and $C^{\dagger}$ are tree of rational curves. Furthermore we assume that relative Picard numbers of $f$ and $g$ are one, and $\phi: W \rightarrow X$ is not an isomorphism. In this case the diagram (5.1) is called a flop (cf. [28]). The main technical tool we use here is the notion of perverse $t$-structures associated to $f: X \rightarrow Y$. It was introduced by T. Bridgeland [8] to construct the derived equivalence between $W$ and $X$. Next we collect some results we need.

Proposition 5.2. There are hearts of bounded t-structures ${ }^{p} \operatorname{Per}\left(\mathcal{D}_{X}\right) \subset$ $\mathcal{D}_{X}$ for $p=-1,0$ which satisfy the following.

(i) For any $E \in{ }^{p} \operatorname{Per}\left(\mathcal{D}_{X}\right)$, we have $\mathbf{R} f_{*} E \in \operatorname{Coh}_{\leq 1}(Y)$.

(ii) There is an equivalence $\Phi: \mathcal{D}_{W} \rightarrow \mathcal{D}_{X}$ which restrict to the equivalence,

$$
\Phi:{ }^{-1} \operatorname{Per}\left(\mathcal{D}_{W}\right) \longrightarrow{ }^{0} \operatorname{Per}\left(\mathcal{D}_{X}\right) .
$$

Furthermore $\Phi$ induce the following commutative diagrams,

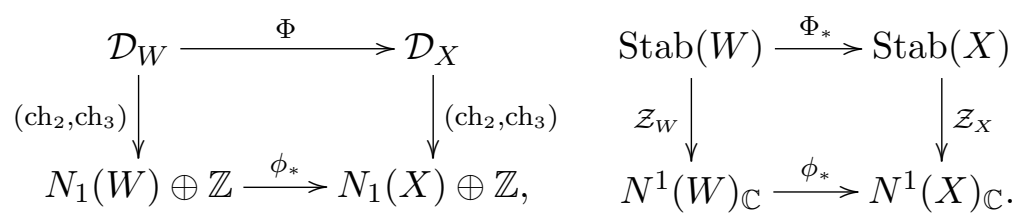

Here $\phi_{*}$ in the left diagram takes $(\beta, k)$ to $\left(\phi_{*} \beta, k\right)$, and $\Phi_{*}$ is the natural isomorphism induced by the equivalence $\Phi$. 
(iii) Let $H$ be a relatively ample divisor on $X$ over $Y$. Then for a sufficiently small $0<\delta \ll 1$ and an ample divisor $\omega^{\prime}$ on $Y$, the pairs

$$
\begin{aligned}
\sigma_{\left(-\delta H, f^{*} \omega^{\prime}\right)} & =\left(Z_{\left(-\delta H, f^{*} \omega^{\prime}\right)},{ }^{0} \operatorname{Per}\left(\mathcal{D}_{X}\right)\right), \\
\sigma_{\left(\delta H, f^{*} \omega^{\prime}\right)} & =\left(Z_{\left(\delta H, f^{*} \omega^{\prime}\right)},{ }^{-1} \operatorname{Per}\left(\mathcal{D}_{X}\right)\right),
\end{aligned}
$$

determine stability conditions contained in $\bar{U}_{X}$.

(iv) For $p=-1,0$, the stack of objects $E \in{ }^{p} \operatorname{Per}\left(\mathcal{D}_{X}\right)$,

$$
{ }^{p} \mathfrak{P e r}(X) \subset \mathcal{M}
$$

is an open substack of $\mathcal{M}$.

Proof. In [8, section 3], Bridgeland constructed the hearts of some bounded $t$-structures ${ }^{p} \operatorname{Per}(X / Y)$ on $D(X)$ for $p=-1,0$. For simplicity we discuss the case of $p=0$. According to $\left[46\right.$, lemma 3.1], the abelian category ${ }^{0} \operatorname{Per}(X / Y)$ is obtained from $\operatorname{Coh}(X)$ as a tilting of the torsion pair,

$$
\begin{aligned}
& \mathcal{T}_{0}=\left\{T \in \operatorname{Coh}(X) \mid R^{1} f_{*} T=0\right\}, \\
& \mathcal{F}_{0}=\left\{F \in \operatorname{Coh}(X) \mid f_{*} F=0, \operatorname{Hom}(\mathfrak{C}, F)=0\right\},
\end{aligned}
$$

where $\mathfrak{C}:=\left\{E \in \operatorname{Coh}(X) \mid \mathbf{R} f_{*} E=0\right\}$, i.e., ${ }^{0} \operatorname{Per}(X / Y)$ is generated by $\mathcal{F}_{0}[1]$ and $\mathcal{T}_{0}$. Let us define ${ }^{p} \operatorname{Per}\left(\mathcal{D}_{X}\right)$ to be

$$
{ }^{p} \operatorname{Per}\left(\mathcal{D}_{X}\right):=\mathcal{D}_{X} \cap{ }^{p} \operatorname{Per}(X / Y) .
$$

We have to check that ${ }^{p} \operatorname{Per}\left(\mathcal{D}_{X}\right)$ is the heart of a bounded $t$-structure on $\mathcal{D}_{X}$. Since $\mathcal{F}_{0} \subset \operatorname{Coh}_{\leq 1}(X)$, the pair $\left(\mathcal{F}_{0}, \mathcal{T}_{0} \cap \mathrm{Coh}_{\leq 1}(X)\right)$ also determines a torsion pair on $\operatorname{Coh}_{\leq 1}(X)$, and the corresponding tilting is ${ }^{0} \operatorname{Per}\left(\mathcal{D}_{X}\right)$. Thus ${ }^{0} \operatorname{Per}\left(\mathcal{D}_{X}\right)$ is the heart of a bounded $t$-structure on $\mathcal{D}_{X}$ (cf. [15]).

(i) For $E \in{ }^{p} \operatorname{Per}\left(\mathcal{D}_{X}\right)$, the object $\mathbf{R} f_{*} E$ must be a sheaf by the definition of ${ }^{p} \operatorname{Per}(X / Y)$ in $[8$, section 3$]$.

(ii) In [8], Bridgeland constructed the equivalence,

$$
\Phi: D(W) \longrightarrow D(X)
$$

which restricts to an equivalence between ${ }^{-1} \operatorname{Per}(W / Y)$ and ${ }^{0} \operatorname{Per}(X / Y)$. Furthermore Chen [9] showed that $\Phi$ is given by a Fourier-Mukai functor with kernel $\mathcal{O}_{W \times_{Y} X}$. Because $\phi: W \rightarrow X$ is an isomorphism in codimension one, the equivalence $\Phi$ takes $\mathcal{D}_{W}$ to $\mathcal{D}_{X}$. 
For the left diagram of (5.2), let us take a divisor $D$ on $X$ and $E \in \mathcal{D}_{W}$. By Riemann-Roch theorem, we have

$$
\begin{aligned}
\chi\left(\mathcal{O}_{X}(D), \Phi(E)\right) & =-D \cdot \operatorname{ch}_{2} \Phi(E)+\operatorname{ch}_{3} \Phi(E), \\
\chi\left(\Phi^{-1} \mathcal{O}_{X}(D), E\right) & =-\phi_{*}^{-1} D \cdot \operatorname{ch}_{2}(E)+\operatorname{ch}_{3}(E) .
\end{aligned}
$$

Here we have used the fact that $\operatorname{ch}_{1} \Phi^{-1} \mathcal{O}_{X}(D)=\phi_{*}^{-1} D$. This follows because $\Phi^{-1}\left(\mathcal{O}_{X}(D)\right)$ and $\mathcal{O}_{X}\left(\phi_{*}^{-1} D\right)$ are isomorphic over $W \backslash C^{\dagger}$, and $C^{\dagger}$ has codimension two in $W$ (cf. [42, lemma 3.15]). By adjunction we must have $(5.4)=(5.5)$, and this holds for any divisor $D$. Thus we have

$$
\left(\operatorname{ch}_{2} \Phi(E), \operatorname{ch}_{3} \Phi(E)\right)=\left(\phi_{*} \operatorname{ch}_{2}(E), \operatorname{ch}_{3}(E)\right)
$$

by the definition of $\phi_{*}: N_{1}(W) \rightarrow N_{1}(X)$.

For the commutativity of the right diagram of (5.2), the same proof of [42, lemma 4.8] is applied, and we leave the readers to check the detail.

(iii) The same proof of [42, lemma 4.3] shows that the pairs (5.3) give stability conditions. In fact arguing as in [42, lemma 3.8 (iii)], any object in ${ }^{0} \operatorname{Per}\left(\mathcal{D}_{X}\right)$ is given by a successive extension of the following objects,

$$
\begin{aligned}
& S_{0}=\omega_{f^{-1}(0)}[1], \quad S_{i}=\mathcal{O}_{C_{i}}(-1)(1 \leq i \leq m), \\
& \widetilde{\operatorname{Coh}}_{\leq 1}(X):=\left\{F \in \operatorname{Coh}_{\leq 1}(X) \mid C_{i} \nsubseteq \operatorname{Supp}(F) \text { for all } i\right\} .
\end{aligned}
$$

Here $C_{i}$ for $1 \leq i \leq m$ are the irreducible components of $C$ and $f^{-1}(0)$ is the scheme theoretic fiber of $f$ at $0 \in Y$. In order to show (3.1) in Proposition 3.2, it is enough to check this for the generators (5.6) and (5.7). For $Z=Z_{\left(-\delta H, f^{*} \omega^{\prime}\right)}$, we have

$$
\begin{aligned}
& Z\left(S_{0}\right)=-1+\delta H \cdot f^{-1}(0)<0, \quad Z\left(S_{i}\right)=-\delta H \cdot C_{i}<0(1 \leq i \leq m), \\
& \operatorname{Im} Z(F)>0 \quad \text { for } F \in \widetilde{\operatorname{Coh}}_{\leq 1}(X) \backslash\{0\},
\end{aligned}
$$

thus (3.1) holds. We leave the readers to check the Harder-Narasimhan property, applying the proof of [42, lemma 4.3]. Also the case of $p=-1$ is similarly proved. Finally we have to check that the stability conditions determined by (5.3) are contained in $\bar{U}_{X}$. Since it requires some more technical arguments, we postpone it until Section 6 . 
(iv) According to [46], there are vector bundles ${ }^{p} \mathcal{E}$ on $X$ for $p=-1,0$ such that an object $E \in \mathcal{D}_{X}$ is contained in ${ }^{p} \operatorname{Per}\left(\mathcal{D}_{X}\right)$ if and only if

$$
\mathbf{R} f_{*} \mathbf{R} \mathcal{H o m}\left({ }^{p} \mathcal{E}, E\right) \in \operatorname{Coh}(Y) .
$$

Since (5.8) is an open condition, the stack ${ }^{p} \mathfrak{P e r}(X)$ is an open substack of $\mathcal{M}$.

For $v=(\beta, k) \in N_{1}(X) \oplus \mathbb{Z}$, let

$$
{ }^{p} \mathfrak{P e r}^{v}(X) \subset{ }^{p} \mathfrak{P e r}(X),
$$

the substack of objects $E \in{ }^{p} \operatorname{Per}\left(\mathcal{D}_{X}\right)$ of numerical type $v$. By Proposition 5.2 (i), we have the 1-morphism,

$$
\bar{\pi}:{ }^{p} \mathfrak{P e r}^{v}(X) \ni E \longmapsto s\left(\mathbf{R} f_{*} E\right) \in \mathrm{Chow}_{f_{*} \beta}(Y)
$$

Let $\sigma \in \bar{U}_{X}$ be one of $(5.3)$, corresponding to ${ }^{p} \operatorname{Per}\left(\mathcal{D}_{X}\right)$ for $p=-1$ or 0 . As in (4.14), for $0<\phi \leq 1$ we have

$$
\epsilon^{(v, \phi)}(\sigma) \in K_{0}\left(\mathrm{St} /{ }^{p} \mathfrak{P e r}(X)\right)
$$

Definition 5.3. Let $\sigma \in \bar{U}_{X}$ be one of (5.3). We define $P(v, \sigma) \in \Lambda$ as follows.

- If $v \in C_{\sigma}(\phi)$ with $0<\phi \leq 1$, we define

$$
P(v, \sigma):=(\mathbb{L}-1) \Upsilon_{\bar{A}}^{\prime}\left(\bar{\pi}_{*} \epsilon^{(v, \phi)}(\sigma)\right),
$$

where $\bar{A}=\operatorname{Chow}_{f_{*} \beta}(Y)$. By $(5.9), \bar{\pi}_{*} \epsilon^{(v, \phi)}(\sigma) \in K_{0}($ St $/ \bar{A})$ makes sense.

- If $v \in C_{\sigma}(\phi)$ with $1<\phi \leq 2$, we define

$$
P(v, \sigma):=P(-v, \sigma)
$$

- Otherwise we define $P(v, \sigma)=0$. 
Remark 5.4. In [46], it is shown that there are vector bundles ${ }^{p} \mathcal{E}$ on $X$ for $p=-1,0$ such that there are equivalences,

$$
{ }^{p} \operatorname{Per}(X / Y) \stackrel{\sim}{\longrightarrow} \operatorname{Coh}\left(f_{*} \mathcal{H} \text { om }\left({ }^{p} \mathcal{E},{ }^{p} \mathcal{E}\right)\right) .
$$

Since the right-hand side is the module category over a non-commutative sheaf of algebras on $Y$, BPS counting constructed from Definition 5.3 and the formula (4.17) is interpreted as (approximation of) non-commutative Gopakumar-Vafa invariant. It seems interesting to pursue its relationship to non-commutative Donaldson-Thomas theory on conifold studied by B. Szendröi [40].

For $\sigma \in \bar{U}_{X}$ as in Proposition 5.2 (iii), let us write it $\sigma=(Z, \mathcal{P})$ as in Definition 3.1. We also use the abelian category

$$
\mathcal{A}_{\sigma}=\mathcal{P}\left(\left(-\frac{1}{2}, \frac{1}{2}\right]\right),
$$

and the stack of objects in $\mathcal{A}_{\sigma}, \mathfrak{O b j}\left(\mathcal{A}_{\sigma}\right) \subset \mathcal{M}$. By Proposition 5.2(iv) and [41, proposition 3.18], $\mathfrak{O} \mathfrak{b j}\left(\mathcal{A}_{\sigma}\right)$ is an open substack of $\mathcal{M}$. We have the following lemma.

Lemma 5.5. Under the previous situation, the following diagram is commutative,

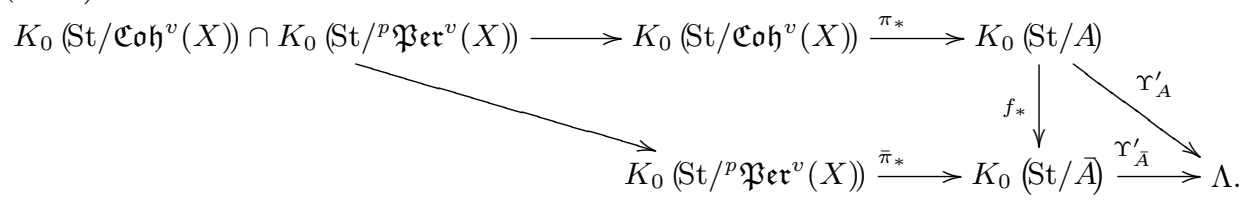

Here $A=\operatorname{Chow}_{\beta}(X)$ and $\bar{A}=\operatorname{Chow}_{f_{*} \beta}(Y)$. Furthermore if $f^{*} \omega^{\prime} \cdot \beta=0$, the following diagram commutes,

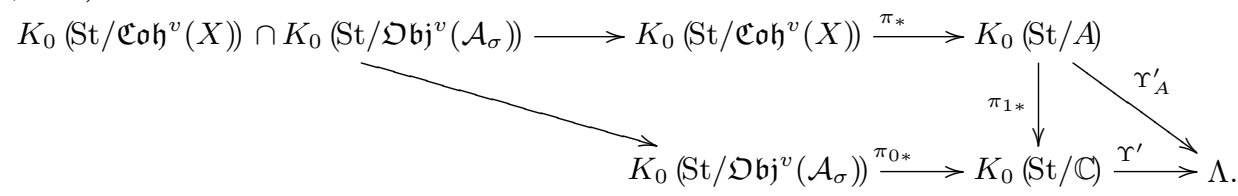


Here $\pi_{0}: \mathfrak{O b j}^{v}\left(\mathcal{A}_{\sigma}\right) \rightarrow$ Spec $\mathbb{C}, \pi_{1}: A \rightarrow$ Spec $\mathbb{C}$ are the structure morphisms. The diagram also commutes after the following replacements.

$$
\begin{aligned}
& \mathfrak{C o h}^{v}(X) \longmapsto \mathfrak{C o h}^{v}(X)[1], \quad \pi \longmapsto \pi^{\prime}, \quad A \longmapsto A^{\prime}, \\
& \mathfrak{C o h}^{v}(X) \longmapsto \operatorname{Per}^{v}(X), \quad \pi \longmapsto \bar{\pi}, \quad A \longmapsto \bar{A} .
\end{aligned}
$$

Here we have used the notation of Remark 4.23.

Proof. In both diagrams, the commutativity of the left-hand side follows from the functorial property of the push-forwards. Let us show the commutativity of the right-hand side. Since $f: X \rightarrow Y$ contracts only finite number of rational curves, the map

$$
f_{*}: A \ni Z \longmapsto f_{*} Z \in \bar{A},
$$

which sends an algebraic cycle $Z$ on $X$ to the cycle $f_{*} Z$ on $Y$, is a finite morphism (see [29, theorem 6.8] for the existence of the above morphism). Hence we can apply Lemma 4.5, which shows the commutativity of the right-hand side of (5.10). Finally if $f^{*} \omega^{\prime} \cdot \beta=0$, any effective one cycle of homology class $\beta$ is contracted by $f$. Thus $\bar{A}=$ Spec $\mathbb{C}$, and $f_{*}: A \rightarrow \bar{A}$ is identified with $\pi_{1}$. Therefore the commutativity of the right-hand side of (5.11) also follows from Lemma 4.5.

Now we show the following proposition.

Proposition 5.6. Let $\sigma \in \bar{U}_{X}$ be as before and $\tau=(W, \mathcal{Q}) \in U_{X}$. Then for any $v \in N_{1}(X) \oplus \mathbb{Z}$, one has $P(v, \sigma)=P(v, \tau)$.

Proof. It is enough to show $P(v, \sigma)=P(v, \tau)$ under the situation of Proposition 4.28. Furthermore the same proof of Proposition 4.30 shows $P(v, \sigma)=$ $P(v, \tau)=0$ if $v \notin C_{\sigma}(\phi)$ for any $\phi \in \mathbb{R}$. Thus we may assume $v \in C_{\sigma}(\phi)$ for some $0<\phi \leq 1$. First we assume $0<\phi<1$. Let us take $\varepsilon>0$ as in Proposition 4.28. We can choose $\varepsilon>0$ sufficiently small so that $0<\phi-2 \varepsilon<$ $\phi+2 \varepsilon<1$. Then we have

$$
\mathcal{Q}((\phi-\varepsilon, \phi+\varepsilon)) \subset{ }^{p} \operatorname{Per}\left(\mathcal{D}_{X}\right) \cap \operatorname{Coh}_{\leq 1}(X) .
$$

Thus all the terms in (4.19) are contained in both $K_{0}\left(\operatorname{St} /{ }^{p} \mathfrak{P e r}(X)\right)$ and $K_{0}(\operatorname{St} / \mathfrak{C o h}(X))$, and (4.20) holds in both algebras. Applying Lemma 4.31 
for $\mathcal{A}=\operatorname{Coh}_{\leq 1}(X)$, we have

$$
(\mathbb{L}-1) \Upsilon_{A}^{\prime}\left(\pi_{*} \epsilon^{(v, \phi)}(\sigma)\right)=(\mathbb{L}-1) \Upsilon_{A}^{\prime}\left(\pi_{*} \epsilon^{(v, \psi)}(\tau)\right),
$$

for some $\psi \in(\phi-\varepsilon, \phi+\varepsilon)$. (Here we have used the same notation as in Lemma 5.5.) By Definition 4.22, the right-hand side of $(5.10)$ is $P(v, \tau)$. On the other hand we have

$$
(\mathbb{L}-1) \Upsilon_{A}^{\prime}\left(\pi_{*} \epsilon^{(v, \phi)}(\sigma)\right)=(\mathbb{L}-1) \Upsilon_{\bar{A}}^{\prime}\left(\bar{\pi}_{*} \epsilon^{(v, \phi)}(\sigma)\right),
$$

by the diagram (5.10). Then the right-hand side of $(5.11)$ is $P(v, \sigma)$ by Definition 5.3. Hence (5.10) and (5.11) show $P(v, \sigma)=P(v, \tau)$.

Next suppose $\phi=1$. Note that in this case $f^{*} \omega^{\prime} \cdot \beta=0$ for $v=(\beta, k)$. For a sufficiently small $\varepsilon>0$, one has

$$
\mathcal{Q}((1-\varepsilon, 1+\varepsilon)) \subset \mathcal{A}_{\sigma}
$$

Thus the formulae (4.19) and (4.20) hold in $K_{0}\left(\mathrm{St} / \mathfrak{O b j}\left(\mathcal{A}_{\sigma}\right)\right)$. Applying Lemma 4.31 for $\mathcal{A}=\mathcal{A}_{\sigma}$, we have

$$
(\mathbb{L}-1) \Upsilon^{\prime}\left(\pi_{0 *} \epsilon^{(v, 1)}(\sigma)\right)=(\mathbb{L}-1) \Upsilon^{\prime}\left(\pi_{0 *} \epsilon^{(v, \psi)}(\tau)\right),
$$

for some $\psi \in(1-\varepsilon, 1+\varepsilon)$. If $\psi \leq 1$, then $\epsilon^{(v, \psi)}(\tau)$ is contained in both $K_{0}(\mathrm{St} / \mathfrak{C o h}(X))$ and $K_{0}\left(\mathrm{St} / \mathfrak{O b j}\left(\mathcal{A}_{\sigma}\right)\right)$, hence the right-hand side of $(5.12)$ is equal to $P(v, \tau)$ by the diagram (5.11). Also the diagram (5.11) after the following replacement,

$$
\mathfrak{C o h}^{v}(X) \longmapsto{ }^{p} \mathfrak{P e r}^{v}(X), \quad \pi \longmapsto \bar{\pi} \quad A \longmapsto \bar{A},
$$

shows that the left-hand side of (5.12) is equal to $P(v, \sigma)$. Hence in this case we obtain $P(v, \sigma)=P(v, \tau)$. When $\psi>1$, we can use the diagram (5.12) after the replacement,

$$
\mathfrak{C o h}^{v}(X) \longmapsto \mathfrak{C o h}^{v}(X)[1], \quad \pi \longmapsto \pi^{\prime} \quad A \longmapsto A^{\prime},
$$

and conclude that $P(v, \sigma)=P(v, \tau)$. In fact by Remark 4.23, we see that the right-hand side of $(5.12)$ is equal to $P(v, \tau)$ also in this case.

\subsection{Proof of Theorem 5.1}

Proof. We may assume that there is a diagram (5.1), since any birational map $\phi: W \rightarrow X$ is connected by a sequence of flops [27]. Let $H$ be a 
relatively ample divisor on $W$ over $Y$ and

$$
\sigma=\left(Z_{\left(-\delta H, g^{*} \omega^{\prime}\right)}, \quad{ }^{-1} \operatorname{Per}\left(\mathcal{D}_{W}\right)\right) \in \bar{U}_{W}
$$

the stability condition in (5.3), applied for $g: W \rightarrow Y$. By Proposition 5.2 (ii), we have

$$
\Phi_{*} \sigma=\left(Z_{\left(-\delta \phi_{*} H, f^{*} \omega^{\prime}\right)}, \quad{ }^{0} \operatorname{Per}\left(\mathcal{D}_{X}\right)\right)
$$

(Here we have used the right diagram of (5.2).) Since $-\phi_{*} H$ is relatively ample over $Y, \Phi_{*} \sigma$ is one of the stability conditions constructed in (5.3). Hence by Proposition 5.6, it is enough to show

$$
P(W, v, \sigma)=P\left(X, \phi_{*} v, \Phi_{*} \sigma\right)
$$

We may assume $v \in C_{\sigma}(\phi)$ for $0<\phi \leq 1$. By Proposition 5.2 (ii), the equivalence $\Phi$ induces the isomorphism,

$$
\Phi_{\mathrm{St}}: K_{0}\left(\mathrm{St} /{ }^{-1} \mathfrak{P e r}(W)\right) \longrightarrow K_{0}\left(\mathrm{St} /{ }^{0} \mathfrak{P e r}(X)\right)
$$

It is easy to see that (5.14) preserves $*$-product. Furthermore by the left diagram of (5.2), the isomorphism (5.14) takes $\delta^{(v, \phi)}(\sigma)$ to $\delta^{\left(\phi_{*} v, \phi\right)}\left(\Phi_{*} \sigma\right)$. Thus we have

$$
\Phi_{\mathrm{St}} \epsilon^{(v, \phi)}(\sigma)=\epsilon^{\left(\phi_{*} v, \phi\right)}\left(\Phi_{*} \sigma\right)
$$

On the other hand, we have the commutative diagram,

$$
K_{0}\left(\mathrm{St} /{ }^{-1} \operatorname{Per}^{v}(W)\right) \stackrel{\Phi_{\mathrm{St}}}{\longrightarrow} K_{0}\left(\mathrm{St} /{ }^{0} \mathfrak{P e r}^{\phi_{*} v}(X)\right)
$$

Hence the diagram (5.16) together with (5.15) imply (5.13).

Remark 5.7. If $v=(0,1)$ and $\sigma \in U_{X}$, then $P(v, \sigma)$ is equal to $\sum_{i} b_{i}(X) t^{i}$. Hence Proposition 5.6 and (5.13) imply $b_{i}(W)=b_{i}(X)$ for all $i \in \mathbb{Z}$.

Example 5.8. Let $\mathbb{P}^{1} \cong C \subset X$ be a $(-1,-1)$ curve, i.e., the normal bundle $N_{C / X}$ is isomorphic to $\mathcal{O}_{C}(-1) \oplus \mathcal{O}_{C}(-1)$. For $m \neq 0$, the same 
computation in [16, proposition 4.5] shows,

$$
n_{g}^{m[C]}(X)= \begin{cases}1 & \text { if } g=0, m= \pm 1 \\ 0 & \text { otherwise }\end{cases}
$$

Let $\phi: W \rightarrow X$ be a flop at $C$, and $C^{\dagger} \subset W$ the flopped curve. Since $\phi_{*}\left[C^{\dagger}\right]=-[C]$, one can see

$$
n_{g}^{\left[C^{\dagger}\right]}(W)=n_{g}^{-[C]}(X)=1
$$

by the computation shown. Note that since $-[C]$ is not effective, the invariant $n_{g}^{\beta}(X)$ should also be defined for non-effective one cycle classes $\beta$.

\section{Some technical lemmas}

In this section, we prove some postponed technical lemmas.

\subsection{Proof of Lemma 3.6}

Let us take $\sigma=\sigma_{(B, \omega)} \in U_{X}$ and $B^{\prime}+i \omega^{\prime} \in N^{1}(X)_{\mathbb{C}}$. First we show $\| Z_{(B, \omega)}$ $-Z_{\left(B^{\prime}, \omega^{\prime}\right)} \|_{\sigma}<\infty$ (see $\left[7\right.$, section 6] for $\left.\|*\|_{\sigma}\right)$. By the definition of $\|*\|_{\sigma}$, it is equal to

$$
\sup \left\{\frac{\left|\left\{\left(B-B^{\prime}\right)+i\left(\omega-\omega^{\prime}\right)\right\} \operatorname{ch}_{2}(E)\right|}{\left|Z_{(B, \omega)}(E)\right|}: E \text { is semistable in } \sigma\right\} \text {. }
$$

Let us put $m=\left|Z_{(B, \omega)}(E)\right|$ for a $\sigma$-semistable object $E$. Then we have $\left|\omega \cdot \operatorname{ch}_{2}(E) / m\right| \leq 1$. We set $K \subset N_{1}(X)$ as

$$
K:=\{c \in \overline{\mathrm{NE}}(X) \mid \omega \cdot c \leq 1\} \subset N_{1}(X) .
$$

As in the proof of Proposition 3.7, the space $K$ is compact. Therefore the function

$$
K \ni c \longmapsto\left|\left\{\left(B-B^{\prime}\right)+i\left(\omega-\omega^{\prime}\right)\right\} c\right| \in \mathbb{R}
$$

has a maximum value, say $M$. Since $\operatorname{ch}_{2}(E) / m \in K$ or $-\operatorname{ch}_{2}(E) / m \in K$, we have $(6.1) \leq M<\infty$. 
Then by [7, proposition 6.3], the map $\operatorname{Stab}(X) \rightarrow N^{1}(X)_{\mathbb{C}}$ is a local homeomorphism. Suppose that $B^{\prime}+i \omega^{\prime}$ satisfies

$$
\left\|Z_{(B, \omega)}-Z_{\left(B^{\prime}, \omega^{\prime}\right)}\right\|_{\sigma}<\sin \pi \varepsilon
$$

for a sufficiently small $\varepsilon$. Then [7, theorem 7.1] guarantees the existence of a stability condition $\tau=\left(Z_{\left(B^{\prime}, \omega^{\prime}\right)}, \mathcal{Q}\right)$ which satisfies $d(\mathcal{P}, \mathcal{Q})<\varepsilon$ (see $[7$, section 6$]$ for $d(*, *))$. If we know $\tau \in U_{X}$, we can conclude $U_{X}$ is open.

To conclude $\tau \in U_{X}$, it is enough to check $\mathcal{Q}((0,1]) \subset \operatorname{Coh}_{\leq 1}(X)$. According to the proof of [7, theorem 7.1], the set of objects $\mathcal{Q}(\phi)$ for $0<\phi \leq 1$ is obtained as follows: an object $E \in \mathcal{D}_{X}$ is contained in $\mathcal{Q}(\phi)$ if and only if there is a thin and enveloping subcategory $E \in \mathcal{P}((a, b))$ such that $E$ is $Z_{\left(B^{\prime}, \omega^{\prime}\right)}$-semistable with phase $\phi$ (see [7, definitions 7.2 and 7.4] for the notion of thin enveloping subcategory). Take $E \in \mathcal{Q}(\phi)$ with $E \in \mathcal{P}((a, b))$ as before. If $0<a<b \leq 1$, one has $E \in \mathrm{Coh}_{\leq 1}(X)$. Suppose $b>1$. Then there is a distinguished triangle

$$
H^{-1}(E)[1] \longrightarrow E \longrightarrow H^{0}(E)
$$

with $H^{-1}(E)[1] \in \mathcal{P}((1, b))$ and $H^{0}(E) \in \mathcal{P}((a, 1])$. The semistability of $E$ in $Z_{\left(B^{\prime}, \omega^{\prime}\right)}$ implies

$$
\arg Z_{\left(B^{\prime}, \omega^{\prime}\right)}\left(H^{-1}(E)[1]\right) \leq \arg Z_{\left(B^{\prime}, \omega^{\prime}\right)}\left(H^{0}(E)\right) .
$$

Here arg is taken in the interval $(\pi i(a-\varepsilon), \pi i(b+\varepsilon))$. However since $H^{i}(E)$ $\in \mathrm{Coh}_{\leq 1}(X)$, (6.4) implies $E \cong H^{0}(E)$ or $E \cong H^{-1}(E)[1]$. Since $E$ has phase $0<\phi \leq 1$ with respect to $Z_{\left(B^{\prime}, \omega^{\prime}\right)}$, one must have $E \cong H^{0}(E) \in$ $\mathrm{Coh}_{\leq 1}(X)$. The similar argument shows $E \in \mathrm{Coh}_{\leq 1}(X)$ when $a \leq 0$.

\subsection{Proof of Lemma 3.8}

In fact we show the following stronger claim. Let $\Sigma \subset \operatorname{Stab}\left(\mathcal{D}_{X}\right)$ be the connected component which contains $U_{X}$, and $\Sigma^{\prime} \subset \Sigma$ the subset of $\sigma=$ $(Z, \mathcal{P})$ such that $\operatorname{Im} Z \subset \mathbb{C}$ is discrete. We show that for any $\sigma \in \Sigma^{\prime}, \phi \in \mathbb{R}$ and $z \in \mathbb{C}$, the following set of objects,

$$
M^{(z, \phi)}(\sigma):=\{E \in \mathcal{P}(\phi) \mid Z(E)=z\}
$$

is bounded. For an ample divisor $\omega$ on $X$, let us consider the point $\sigma_{(0, \omega)} \in$ $U_{X}$. By Remark 3.5, any $\sigma_{(0, \omega)}$-semistable object is nothing but $\omega$-Gieseker semistable sheaf up to shift. As is well known, the set of $\omega$-Gieseker 
semistable sheaves with a fixed Hilbert polynomial forms a bounded family, hence the claim is true for $\sigma=\sigma_{(0, \omega)}$.

Next suppose that the previous claim is true for some $\sigma=(Z, \mathcal{P}) \in \Sigma^{\prime}$. We show that if $\tau=(W, \mathcal{Q}) \in \Sigma^{\prime}$ is sufficiently close to $\sigma$, then the claim also holds for $\tau$. Obviously once we show this, then the claim is true for any $\sigma \in \Sigma^{\prime}$. In order to show the boundedness of $M^{(z, \phi)}(\tau)$, we may assume $\phi=1 / 2$ by applying some element $g \in \widetilde{\mathrm{GL}}^{+}(2, \mathbb{R})$ (cf. [7, lemma 8.2]) to $\sigma$, $\tau$. If $\tau$ is sufficiently close to $\sigma$, we have

$$
\mathcal{Q}\left(\frac{1}{2}\right) \subset \mathcal{P}\left(\left(\frac{1}{4}, \frac{3}{4}\right)\right) \subset \mathcal{Q}((0,1)) .
$$

For $E \in M^{(z, 1 / 2)}(\tau)$, let $F_{i} \in \mathcal{P}\left(\phi_{i}\right)$ for $\phi_{i} \in(1 / 4,3 / 4), 1 \leq i \leq n$ be the $\sigma$ semistable factors of $E$. Since $\operatorname{Im} W\left(F_{i}\right) \leq \operatorname{Im} W(E)$, there is $\delta>0$ which does not depend on $E$ such that $\operatorname{Im} Z\left(F_{i}\right) \leq \operatorname{Im} z+\delta$, if $\tau$ is enough close to $\sigma$. Because $\operatorname{Im} Z \subset \mathbb{C}$ is discrete, we see that numbers of semistable factors $n$, and the values $z_{i}=Z\left(F_{i}\right) \in \mathbb{C}$ have finite number of possibilities. Since $F_{i} \in M^{\left(z_{i}, \phi_{i}\right)}(\sigma)$, the boundedness of $M^{\left(z_{i}, \phi_{i}\right)}(\sigma)$ for each $i$ implies the boundedness of $M^{(z, \phi)}(\tau)$.

\subsection{Proof of Lemma 4.19}

We may assume $0<\phi \leq 1$ and let us take $0<\varepsilon<1 / 6$. Since $\sigma \in \bar{U}_{X}$, there is $\tau=\left(Z_{(B, \omega)}, \operatorname{Coh}_{\leq 1}(X)\right) \in U_{X}$ with $B, \omega$ rational such that $C_{\sigma}(\phi) \subset$ $C_{\tau}((\phi-\varepsilon, \phi+\varepsilon))$. From this it is clear that there is a finite number of possibilities for $n$ in (4.13). Hence it is enough to check the finiteness of the set,

$$
\left\{\left(v_{1}, v_{2}\right) \mid v_{1}+v_{2}=v, v_{i} \in C_{\sigma}(\phi)\right\} .
$$

We write $v_{i}=\left(\beta_{i}, k_{i}\right) \in N_{1}(X) \oplus \mathbb{Z}$. It is enough to check that the possible pairs $\left(\beta_{1}, \beta_{2}\right)$ are finite. First we assume $0<\phi<1$. We may assume that $0<\phi-\varepsilon<\phi+\varepsilon<1$. Then $\beta_{i} \in \overline{N E}(X)$ and we have

$$
\operatorname{Im} Z_{(B, \omega)}\left(v_{i}\right) \leq \operatorname{Im} Z_{(B, \omega)}(v) .
$$

Since (6.5) implies $\beta_{i} \cdot \omega \leq \beta \cdot \omega$ and (6.2) is compact, the possible pairs $\left(\beta_{1}, \beta_{2}\right)$ must be finite.

Next we treat the case of $\phi=1$. Then $v_{i}$ is decomposed as follows,

$$
v_{i}=\sum_{j} v_{i j}, \quad v_{i j} \in C_{\tau}\left(\phi_{i j}\right) \text { with } \phi_{i j} \in(1-\varepsilon, 1+\varepsilon) .
$$


If we write $v_{i j}=\left(\beta_{i j}, k_{i j}\right)$, then $\beta_{i j} \in \overline{N E}(X)$ or $-\beta_{i j} \in \overline{N E}(X)$. We can easily see,

$$
\left|\beta_{i j} \cdot \omega\right|=\left|\operatorname{Im} Z_{(B, \omega)}\left(v_{i j}\right)\right| \leq\left|\operatorname{Re} Z_{(B, \omega)}(v)\right| \cdot \tan \pi \varepsilon .
$$

Again since (6.2) is compact, the possible $\left\{\beta_{i j}\right\}_{i, j}$ are finite. Thus the pair $\left(\beta_{1}, \beta_{2}\right)$ also has a finite number of possibilities.

\subsection{Proof of Proposition 5.2 (iii)}

We have to show stability conditions in (5.3) are contained in $\bar{U}_{X}$. In $[42$, proposition 4.4], the author put the assumption that there exists a hyperplane $Y_{0} \subset Y$ such that $f^{-1}\left(Y_{0}\right)$ is smooth. In our purpose, we have to improve the proof and show that actually stability conditions in (5.3) are contained in $\bar{U}_{X}$ without such assumption.

The proof goes on as in Lemma 3.6, and we show the case of $p=0$ for simplicity. Let $\sigma=\sigma_{\left(-\delta H, f^{*} \omega^{\prime}\right)}$ be as in (5.3), and take $B+i \omega \in N^{1}(X)_{\mathbb{C}}$. We also set $Z=Z_{\left(-\delta H, f^{*} \omega^{\prime}\right)}$. The value $\left\|Z-Z_{(B, \omega)}\right\|_{\sigma}$ is given by

$$
\sup \left\{\frac{\left|\left\{(-\delta H-B)+i\left(f^{*} \omega^{\prime}-\omega\right)\right\} \operatorname{ch}_{2}(E)\right|}{|Z(E)|}: E \text { is semistable in } \sigma\right\} \text {. }
$$

In order to show (6.6) is finite, it is enough to give the upper bound of (6.6) for $E \in{ }^{0} \operatorname{Per}\left(\mathcal{D}_{X}\right)$. Let us take $F \in \widetilde{\operatorname{Coh}}_{\leq 1}(X)$ where $\widetilde{\operatorname{Coh}}_{\leq 1}(X)$ is given by (5.7), and put $m=|Z(F)|$. Then $f^{*} \omega^{\prime} \cdot \operatorname{ch}_{2}(F) / m \leq 1$. By the openness of the big cone, there is a sufficiently small rational polyhedral cone $f^{*} \omega^{\prime} \in \Delta \subset \phi_{*} \bar{A}(W) \cup \bar{A}(X)$. Let $K^{\prime}$ be

$$
K^{\prime}:=\left\{c \in \check{\Delta} \mid f^{*} \omega^{\prime} \cdot c \leq 1\right\} \subset N_{1}(X),
$$

where $\check{\Delta}$ is the dual cone. Then $K^{\prime}$ is compact, hence the function

$$
K^{\prime} \ni c \longmapsto\left|\left\{(-\delta H-B)+i\left(f^{*} \omega^{\prime}-\omega\right)\right\} \cdot c\right| \in \mathbb{R}
$$

has a maximum value, say $M^{\prime}$. Since $F \in \widetilde{\operatorname{Coh}}_{\leq 1}(X)$, we have $\operatorname{ch}_{2}(F)$. $\phi_{*} H^{\prime} \geq 0$, where $H^{\prime}$ is an ample divisor on $W$. Hence $\operatorname{ch}_{2}(F) / m \in K^{\prime}$, which implies

$$
\frac{\left|\left\{(-\delta H-B)+i\left(f^{*} \omega^{\prime}-\omega\right)\right\} \operatorname{ch}_{2}(F)\right|}{|Z(F)|} \leq M^{\prime}
$$

for all $F \in \widetilde{\operatorname{Coh}}_{\leq 1}(X)$. 
Next let us take a non-zero $G \in{ }^{0} \operatorname{Per}\left(\mathcal{D}_{X}\right)$ supported on $C$. Since $G$ is generated by (5.6), we can write $[G]=\sum_{i=1}^{n} a_{i}\left[S_{i}\right]$ in $K\left(\mathcal{D}_{X}\right)$ for $a_{i} \geq 0$. Let us set $c_{i}:=\delta H \cdot C_{i}$ and $c_{i}^{\prime}:=\left|((-\delta H-B)-i \omega) \cdot C_{i}\right|$. We have

$$
\frac{\left|\left\{(-\delta H-B)+i\left(f^{*} \omega^{\prime}-\omega\right)\right\} \mathrm{ch}_{2}(G)\right|}{|Z(G)|} \leq \frac{\sum_{i=1}^{n} a_{i} c_{i}^{\prime}}{a_{0}+\sum_{i=1}^{n} a_{i} c_{i}} .
$$

Since $c_{i}>0$ and $a_{i}>0$ for some $i$, we have RHS $\leq M^{\prime \prime}$ for some $M^{\prime \prime}>0$ independent of $a_{i}$. We may take $M^{\prime \prime}=M^{\prime}$.

Finally since ${ }^{0} \operatorname{Per}\left(\mathcal{D}_{X}\right)$ is generated by (5.6) and (5.7), any $E \in{ }^{0} \operatorname{Per}\left(\mathcal{D}_{X}\right)$ is written as $[E]=[F]+[G]$ in $K\left(\mathcal{D}_{X}\right)$, where $F \in \widehat{\operatorname{Coh}}_{\leq 1}(X)$ and $[G]=$ $\sum_{i=1}^{n} a_{i}\left[S_{i}\right]$ for $a_{i} \geq 0$. We have

$$
\begin{aligned}
(6.6) & \leq \sup \left\{M^{\prime} \cdot \frac{|Z(F)|+|Z(G)|}{|Z(F)+Z(G)|}: E \in{ }^{0} \operatorname{Per}\left(\mathcal{D}_{X}\right)\right\} \\
& \leq M^{\prime} \cdot \sup \left\{\frac{|z|+1}{|z+1|}: \operatorname{Im} z \geq 1\right\}<\infty .
\end{aligned}
$$

Now we have proved $\left\|Z-Z_{(B, \omega)}\right\|_{\sigma}<\infty$. As in the proof of Lemma 3.6, for any $\varepsilon>0$ there is $B+i \omega \in A(X)_{\mathbb{C}}$ and a stability condition $\tau=\left(Z_{(B, \omega)}, \mathcal{Q}\right)$ such that

$$
d(\sigma, \tau)<\varepsilon, \quad\left\|Z-Z_{(B, \omega)}\right\|_{\sigma}<\sin \pi \epsilon .
$$

If we show $\tau \in U_{X}$, we can conclude $\sigma \in \bar{U}_{X}$. The same proof of the last part of Lemma 3.6 shows $\tau \in U_{X}$ (it is enough to notice that in the sequence (6.3), one has $H^{-1}(E)[1] \in \mathcal{P}([1, b))$ and the rest is the same), and we leave the detail to the reader.

\section{Acknowledgments}

This paper was written while the author was visiting the Max-Planck Institut für Mathematik in Bonn. He thanks the institute for the hospitality. Also he thanks Atsushi Takahashi for valuable discussions on GopakumarVafa invariants, Yukiko Konishi for pointing out some references, and Hokuto Uehara, So Okada for giving him good comments on the manuscript. Finally he thanks the referee for many suggestions and comments for the improvement of this paper. He is supported by Japan Society for the Promotion of Sciences Research Fellowships for Young Scientists, no. 198007. 


\section{References}

[1] V.V. Batyrev, Birational Calabi-Yau n-folds have equal Betti numbers, in New Trends in Algebraic Geometry (Warwick,1996), Cambridge University Press, Cambridge, 1999, 1-11.

[2] V.V. Batyrev, Non-Archimedean integrals and stringy Euler numbers of log-terminal pairs, J. Eur. Math. Soc. (JEMS) 1 (1999), 5-33.

[3] K. Behrend, Donaldson-Thomas invariants via microlocal geometry, Preprint, math.AG/0507523.

[4] A. Beilinson, J. Bernstein and P. Deligne, Faisceaux pervers, Anal. Topol. Singular Spaces I, Asterisque 100 (1982), 5-171.

[5] F. Bittner, The universal Euler characteristic for varieties of characteristic zero, Composit. Math. 140 (2004), 1011-1032.

[6] T. Bridgeland, Stability conditions on K3 surfaces, Duke. Math. J., 141 (2008), 241-291.

[7] T. Bridgeland, Stability conditions on triangulated categories, Ann. of Math., 166 (2007), 317-345.

[8] T. Bridgeland, Flops and derived categories, Invent. Math. 147 (2002), 613-632.

[9] J.-C. Chen, Flops and equivalences of derived categories for threefolds with only Gorenstein singularities, J. Differen. Geom. 61 (2002), 227-261.

[10] J. Denef and F. Loeser, Motivic integration, quotient singularities and the McKay correspondence, Composit. Math. 131 (2002), 267-290.

[11] M. Douglas, D-branes, categories and $N=1$ supersymmetry, J. Math. Phys. 42 (2001), 2818-2843.

[12] M. Douglas, Dirichlet branes, homological mirror symmetry, and stability, Proceedings of the 1998 ICM, 2002, 395-408, math.AG/ 0207021 .

[13] R. Gopakumar and C. Vafa, M-theory and topological strings II, hepth/9812127.

[14] P. Griffiths and J. Harris, Principles of Algebraic Geometry, Wiley, New York, 1978. 
[15] D. Happel, I. Reiten and S.O. Smalø, Tilting in abelian categories and quasitilted algebras, Mem. Amer. Math. Soc. 120, 1996.

[16] S. Hosono, M. Saito and A. Takahashi, Relative Lefschetz actions and BPS state counting, Int. Math. Res. Notices. 15 (2001), 783-816.

[17] J. Hu and W.P. Li, The Donaldson-Thomas invariants under blowups and flops, Preprint, math.AG/0505542.

[18] D. Huybrechts and M. Lehn, Geometry of moduli spaces of sheaves, E31, Aspects in Mathematics, Vieweg, 1997.

[19] T. Ito, Stringy Hodge numbers and p-adic Hodge theory, Composit. Math. 140 (2004), 1499-1517.

[20] D. Joyce, Configurations in abelian categories I. Basic properties and moduli stack, Adv. Math. 203 (2006), 194-255.

[21] D. Joyce, Configurations in abelian categories II. Ringel-Hall algebras, Adv. Math. 210 (2007), 635-706.

[22] D. Joyce, Configurations in abelian categories III. Stability conditions and identities, Adv. Math. 215 (2007), 153-219.

[23] D. Joyce, Configurations in abelian categories IV. Invariants and changing stability conditions, Adv. Math. 217 (2008), 125-204.

[24] D. Joyce, Motivic invariants of Artin stacks and 'stack functions', Q. J. Math. 58 (2007), 345-392, math.AG/0509722.

[25] D. Joyce, Holomorphic generating functions for invariants counting coherent sheaves on Calabi-Yau 3-folds, Geom. Topol. 11 (2007), $667-725$.

[26] S. Katz, A. Klemm and C. Vafa, Topological strings and spinning black holes, Adv. Theor. Math. Phys. 3 (1999), 1445-1537.

[27] Y. Kawamata, Flops connect minimal models, Preprint, math.AG/ 0704.1013 .

[28] J. Kollár, Flops, Nagoya Math. J. 113 (1989), 15-36.

[29] J. Kollár, Rational curves on algebraic varieties, 32, Ergebnisse Math. Grenzgeb. (3), Springer-Verlag, Berlin, 1996. 
[30] J. Kollár and S. Mori, Birational geometry of algebraic varieties, 134, Cambridge Tracts in Mathematics, Cambridge University Press, Cambridge, 1998.

[31] Y. Konishi and S. Minabe, Flop invariance of the topological vertex, Preprint, math.AG/0601352.

[32] M. Kontsevich, Lectures at Orsay, 1995.

[33] A.-M. Li and Y. Ruan, Symplectic surgery and Gromov-Witten invariants of Calabi-Yau 3-folds, Invent. Math. 145 (2001), 151-218.

[34] M. Lieblich, Moduli of complexes on a proper morphism, J. Algebr. Geom. 15 (2006), 175-206.

[35] C.H. Liu and S.T. Yau, Transformations of algebraic Gromov-Witten invariants of three-folds under flops and small extremal transitions, with an appendix from the stringy and the symplectic viewpoint, Preprint, math.AG/0505084.

[36] D.R. Morrison, Beyond the Kähler cone, Proceedings of the Hirzebruch 65 Conference on Algebraic Geometry (Ramat Gan, 1993), 361-376.

[37] D. Mumford, J. Fogarty and F. Kirwan, Geometric Invariant Theory: Third Enlarged Edition, Springer-Verlag, Berlin, 1994.

[38] R. Pandharipande and R.P. Thomas, Curve counting via stable pairs in the derived category, Preprint, math.AG/0707.2348.

[39] A. Schwarz and I. Shapiro. Some remarks on Gopakumar-Vafa invariants, Pure. Appl. Math. Q. 1 (2005), 817-826.

[40] B. Szendröi, Non-commutative Donaldson-Thomas theory and the conifold, Preprint, math.AG/0705.3419.

[41] Y. Toda, Moduli stacks and invariants of semistable objects on K3 surfaces, Adv. Math., 217 (2008), 2736-2781.

[42] Y. Toda, Stability conditions and crepant small resolutions, Trans. Am. Math. Soc., to appear, math.AG/0512648.

[43] B. Toën, Higher and derived stacks, Preprint, math.AG/0604504.

[44] B. Toën, Derived Hall algebras, Duke Math. J. 135 (2006), 587-615. 
[45] B. Toën and M. Vaquié, Moduli of objects in dg-categories, Preprint, math.AG/0503269.

[46] M. Van den Bergh, Three dimensional flops and noncommutative rings, Duke Math. J. 122 (2004), 423-455.

[47] C.-L. Wang, On the topology of birational minimal models, J. Different. Geom. 50 (1998), 129-146.

[48] T. Yasuda, Twisted jets, motivic measures and orbifold cohomology, Composit. Math. 140 (2003), 396-422.

Graduate School of Mathematical Sciences

UNIVERSITY OF TOKYO

TOKYO

JAPAN

E-mail address: toda@ms.u-tokyo.ac.jp

RECEIVED JANUARY 25, 2008 